PAPER

Polarized emission in II-VI and perovskite colloidal quantum dots

To cite this article: Maya Isarov et al 2017 J. Phys. B: At. Mol. Opt. Phys. 50214001
Related content

Supplementary data

Topical Review

M Holub and $\mathrm{P}$ Bhattacharya

Optical orientation of excitons and carriers $\frac{\text { in quantum dots }}{\text { Yu G Kusrayev }}$

View the article online for updates and enhancements. 


\title{
Polarized emission in II-VI and perovskite colloidal quantum dots
}

\author{
Maya Isarov ${ }^{1,6}$, Liang $\mathbf{Z} \operatorname{Tan}^{2,6}$, Jenya Tilchin ${ }^{1,6}$, Freddy T Rabouw ${ }^{3,6}$, \\ Maryna I Bodnarchuk ${ }^{4,5}$, Relinde J A van Dijk-Moes ${ }^{3}$, Rotem Carmi ${ }^{1}$, \\ Yahel Barak ${ }^{1}$, Alyssa Kostadinov ${ }^{1}$, Itay Meir ${ }^{1}$, Daniel Vanmaekelbergh ${ }^{3}$, \\ Maksym V Kovalenko ${ }^{4,5}$, Andrew M Rappe ${ }^{2}$ and Efrat Lifshitz ${ }^{1,7}$ \\ ${ }^{1}$ Schulich Faculty of Chemistry, Solid State Institute, Russell Berrie Nanotechnology Institute, Technion, \\ Haifa 3200003, Israel \\ ${ }^{2}$ Department of Chemistry, University of Pennsylvania, Philadelphia, PA 19104-6323, United States of \\ America \\ ${ }^{3}$ Debye Institute of Nanomaterials Science, University of Utrecht, Utrecht, The Netherlands \\ ${ }^{4}$ Institute of Inorganic Chemistry, Department of Chemistry and Applied Biosciences, ETH Zürich, \\ Vladimir Prelog Weg 1, CH-8093 Zürich, Switzerland \\ ${ }^{5}$ Laboratory for Thin Films and Photovoltaics, Empa-Swiss Federal Laboratories for Materials Science \\ and Technology, Überlandstrasse 129, CH-8600 Dübendorf, Switzerland \\ E-mail: ssefrat@technion.ac.il
}

Received 4 July 2017, revised 24 August 2017

Accepted for publication 20 September 2017

Published 23 October 2017

\begin{abstract}
The polarized emission of colloidal quantum dots from II-VI and perovskite semiconductors were investigated thoroughly, revealing information about the optical transitions in these materials and their potential use in various opto-electronic or spintronic applications. The studies included recording of the micro-photoluminescence of individual nanostructures at cryogenic temperatures, with or without the influence of an external magnetic field. The experimental conditions enabled detection of circular and/or linear polarized emission to elucidate the exciton manifolds, angular momentum of the emitting states, Landé $g$-factors, single exciton and biexciton binding energies, the excitons' effective Bohr radii, and the unique influence of the Rashba effect. The study advances the understanding of other phenomena such as electron-hole dissociation, long diffusion lengths, and spin coherence, facilitating appropriate design of optical and spin-based devices.
\end{abstract}

Keywords: colloidal quantum dots, core/shell heterostructures, alloyed interfaces, blinking-free behavior, perovskites, magneto-optical properties, single dot spectroscopy

(Some figures may appear in colour only in the online journal)

Colloidal semiconductor quantum dots (CQDs) are characterized by opto-electronic properties tunable by size and composition [1, 2]. Advanced colloidal synthesis facilitates the formation of high-quality materials with uniform size and morphology and appropriate surface passivation by molecular ligands and/or inorganic shells [3-7]. Fabrication has matured so much that, at present, several nanocrystal families are already used in applications, mostly LEDS for displays

\footnotetext{
6 Equal contribution.

7 Author to whom any correspondence should be addressed.
}

and lighting [8-10], and in the near future, possibly for use in photovoltaic cells [11-13], photo-catalysis [14-16], up-conversion [17, 18], luminescence light concentrator [19], photodetectors [20, 21], biological tagging [22, 23] and spin-based technologies [24, 25]. Along with the development of the synthetic procedures, numerous investigations have explored the optical and electrical properties of these materials, involving the study of exciton emission, electron-hole exchange interaction [26-29], stimulated emission [30-33], multiple exciton generation [34-40], charge injection [41-43], Auger relaxation [44-46], photoconductivity [47], 
and fluorescence intermittency (blinking) [48, 49]. However, spin properties in colloidal nanocrystals have been studied to a lesser extent. Comprehensive understanding of spin polarization could help explain unresolved questions, such as dark/bright state mixing, emanated emission polarization, $g$ factors and spin-spin interactions, all with direct impact on practical applications. The current review focuses on the investigation of exciton polarization in two distinctive materials: (a) II-VI CQDs with core/alloyed-shell or giant-core/ shell structures and a magnetically doped derivative; (b) halide perovskite CQDs. The investigations were carried out by monitoring polarized emission from individual CQDs in a magnetic field. The following sections provide relevant background for each material.

The photon absorption in II-VI CQDs results in the occupation of valence and conduction states by a single electron-hole pair or multiple electron-hole pairs (excitons). For example, in zinc-blende II-VI CQDs, a neutral band-edge single exciton $(X)$ is composed of an electron with spin $S_{\mathrm{e}}=1 / 2$ and a hole with effective spin $J_{\mathrm{h}}=3 / 2$ [26]. The total angular momentum of $X$, denoted as $J_{x}$, can be either 2 or 1 , with $m_{x}= \pm 2, \pm 1,0$. Quantum confinement enhances the electron-hole exchange interaction, which splits the exciton electronic levels into a doublet. There is a further splitting by the crystal field or shape distortions into manifolds of dark and bright states [26]. Under stronger excitation, excess occupation of valence and conduction states occurs, hence charged or multiple excitons (e.g., bi-excitons) are formed, which might also experience degeneracy breaking, depending on their total angular momentum (see below) [50-52].

In recent years, a major concern of the CQDs community is related to occasional fluorescence unsteadiness, manifested as intensity intermittencies (blinking) [48, 49, 53] with consecutive changes from 'on' to 'off', along with spectral diffusion (jittering) [45, 54-56]. Blinking and jittering are the subject of ongoing debates centered around the question of photo-charging generation [49, 57], either by intra-band charge transfer to a remote state (the Auger process) [45] and/or by the charge diffusion to the surface or surrounding [58, 59]. Universal observation of spectral unsteadiness inspired new efforts to achieve photostable materials by controlling the synthesis of the CQDs. This approach has recently led to successful synthesis of materials with unique core/shell architecture, such as core/alloyed-shell [60, 61], core/giant shell, or giant core/shell heterostructures [62-70]. Clearly, spectrally stable CQDs are of prime importance for scientific research and technological applications, and therefore, spectral fluctuations should be mitigated. The giant core/shell CQDs or core/alloyed-shell CQDs are the major material platforms discussed here. These materials consist of a semiconductor core, epitaxially covered by a shell of a second semiconductor compound. The CQD electronic properties depend on the mutual alignment of the core and shell bandedge offsets, which are of type I, type II, or quasi-type II. In type I, the shell band edge wraps that of the core, confining both carriers into the same regime; in type II and in quasitype-II, the core and shell have staggered alignment of the band edges, enabling a complete or a partial charge separation, when one carrier is centered in the core, while the second one is delocalized over the entire core/shell structure [71-75]. The current review describes the properties of the individual $\mathrm{CdTe} / \mathrm{CdTe}_{x} \mathrm{Se}_{1-x}$ core/alloyed-shell and of the $\mathrm{CdTe} / \mathrm{CdSe}$ giant core/shell CQD with different degree of quantum confinement, possessing quasi-type-II and type-I charge distributions, respectively, as shown in figure 1 . As discussed below, the blinking-free behavior of these structures enabled the investigation of polarized transitions related to the formation of single and multiple excitons. The degree of polarization is mainly related to the intrinsic electronic band structure of the studied materials.

Moreover, the review includes a description of how the emission polarization can be significantly enhanced artificially by embedding CQDs with magnetic impurities. Embedding CQDs includes substitution of diamagnetic cations of the host lattice by a single or a few guest magnetic ions, forming diluted magnetic semiconductors (DMS) [76-86]. The most commonly studied cases included incorporation of transition metal ions with valence d-orbitals into II-VI semiconductors. The coupling of magnetic ion d-orbitals with the II-VI semiconductor band-edge states (valence band with p-character and conduction band with $\mathrm{s}$-character) generates $\mathrm{sp}-\mathrm{d}$ exchange interaction [76, 84, 87-89], promoting a strong impact on the magneto-optical properties of the host semiconductors. The CQDs host lattices provide three-dimensional size confinement with a relatively long $(\sim 10 \mathrm{~ns})$ exciton radiative lifetime, which markedly enhances the interaction between resident spins (of the electron, hole or exciton) and the guest spins [90, 91]. A few studies have shown the benefit of magnetically doped CQDs in luminescence solar concentrators or in quantum dots sensitized solar cells. The benefits proceed from the formation of exciton-magnetic polaron, with typical emission that is strongly Stokes shifted from that of the host absorption edge, avoiding re-absorption by neighboring concentrated or sensitized particles [17-19]. A few pioneer groups exploring IIVI DMS-CQDs embedded with $\mathrm{Mn}^{+2}$ and $\mathrm{Cu}^{+2}$ ions [92-96] reported various observations, such as giant Zeeman split [84, 88], circular dichroism [90, 95], magnetic polaron formation [97, 98], fluctuation kinetics [99-101], ion-ion interactions [102, 103] and energy transfer processes [89]. The current review discusses a representative case of $\mathrm{Mn}$ doped in $\mathrm{CdTe} / \mathrm{CdSe}$ CQDs when the guest ions are positioned either in the CdSe core or in the CdS shell.

The halide perovskites have elicited substantial interest since the 1960s [104-106] due to their strong light absorption and emission, high carrier mobility, and apparent high defect tolerance. Interest has revived recently due to groundbreaking discoveries of the perovskites' usefulness in photovoltaic cells, with power conversion efficiencies rivaling other technologies based on organic, Si, CdTe, CIGS and GsAs materials [107-111]. Moreover, the halide perovskites perform well in light emitting diodes [112], lasers [113, 114], photodetectors $[115,116]$ and hold promise as single photon source [117] in spintronics [118]. The renaissance of the halide perovskites also initiated investigations on the microscopic physics underlying the unique performance of these materials 


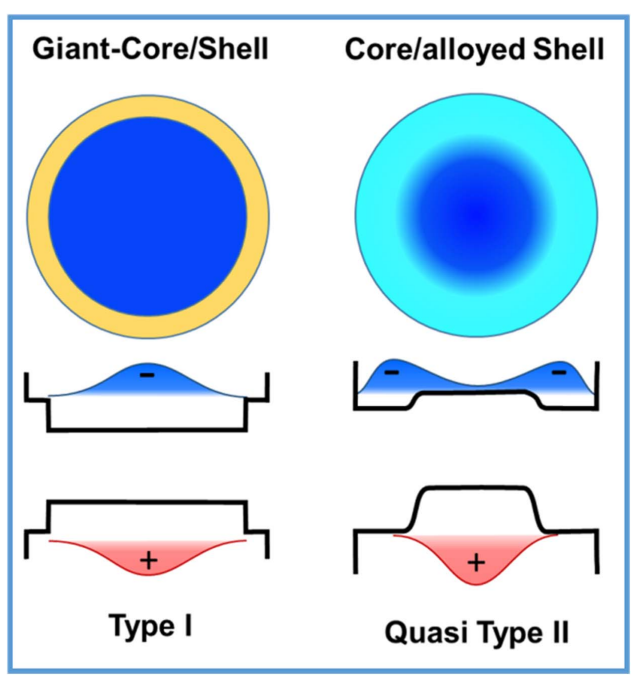

Figure 1. Top: scheme of giant core/shell type-I (left) and core/ alloyed-shell (quasi)type-II CQDs (top). Bottom: anticipated carriers' distribution across the CQD structures shown above.

[119-121]. Serious attention has recently been focused on halide perovskites of the type $\mathrm{AMX}_{3}\left(\mathrm{~A}=\mathrm{Cs}^{+1}\right.$, methyl ammonium [MA], form amidinium [FA]; $\mathrm{M}=\mathrm{Pb}, \mathrm{Ga}, \mathrm{In}$; $\mathrm{X}=\mathrm{I}, \mathrm{Cl}, \mathrm{Br}$ ). They are composed of inter-connected $\mathrm{MX}_{6}^{-4}$ octahedral units, while the 'A' charge balancing species occupies cages created by twelve halide atoms within the octahedral network. The electronic band structure of $\mathrm{AMX}_{3}$ encompasses the $\left[\mathrm{PbX}_{6}\right]$ units; the valence band is composed of $\mathrm{Pb}(6 \mathrm{~s})$ and halide (p) orbitals. The conduction band is mainly compiled from the antibonding $\mathrm{Pb}(6 \mathrm{p})$-halide (p) interactions with a small contribution from halide (s) atomic orbitals $[122,123]$. The $\mathrm{AMX}_{3}$ are known as direct band gap semiconductors, with extrema at the $\mathrm{R}$ [111] Brillouin point. Previous theoretical studies [122, 124] showed a strong influence of spin-orbit coupling at the conduction band, leading to formation of split-off states. Accordingly, the valence and conduction band-edges each have an angular momentum $J=1 / 2$, i.e., the allowed band-edge absorbance is related to the $\pm 1 / 2 \leftrightarrow \pm 1 / 2$ transitions. Furthermore, crystal field symmetry as well as exchange interaction induce additional tuning and elevation of degeneracies, respectively, although the consequent influence of these interactions should be further studied [125]. This review describes current knowledge of the excitons in halide perovskite CQDs, revealing information about band-edge excitons with optical polarization emanated from a spin-orbit effective magnetic field (so-called Rashba effect) [118, 119, 126].

The polarized emission properties in various nanostructures discussed reveal the angular momentum of the emitting states, band-edge electronic properties, electron-hole exchange, host carrier to guest-ion spin-exchange, and spinorbit Rashba interaction. Along with polarized emission detection, other important physical parameters, including effective Landé $g$-factor of the excitons, exciton binding energy and effective Bohr radii and spin-exchange coupling to impurities, have been defined. All parameters are critical when designing devices based on II-VI CQDs or perovskite materials. For example, small binding energy permits an immediate dissociation of an exciton. Polarized and narrow emission lines and coupling to impurity spins are advantageous in designing light sources or spin-based devices.

\section{Results and discussion:}

\section{$\mathrm{CdTe} / \mathrm{CdTe} \mathrm{Se}_{1_{-x}}$ core/alloyed-shell CQDs}

Recent theoretical investigations emphasized the benefit of the alloyed interface between a core and a shell in suppressing an Auger process $[127,128]$. Thus, the $\mathrm{CdTe} / \mathrm{CdTe}_{x} \mathrm{Se}_{1-x}$ core/alloyed-shell CQDs were found to be a good platform for investigating exciton and multiple excitons in CQDs. These CQDs have zinc-blende (space groupF $\overline{4} 3 \mathrm{~m}$ ) crystal structure with tuneable band-edge energy by varying the overall size as well as by the core-to-shell radius ratio. A CQD with core diameter of $3.2 \mathrm{~nm}$ and a shell thickness of $0.6 \mathrm{~nm}$ showing a quasi-type-II band alignment is discussed. The synthesis of $\mathrm{CdTe} / \mathrm{CdTe}_{x} \mathrm{Se}_{1-x}$ core/shell CQDs was based on a similar procedure given in reference [129]. The $\mu$ PL spectra of the sample was recorded by dispersing dots over a quartz substrate with a density $<1 \mathrm{NQD} \mu \mathrm{m}^{-2}$, which were examined by the use of fiber-based confocal microscope, together incorporated in a cryo-magnetic system. The samples were pumped by a continuous-wave $514.5 \mathrm{~nm}$ line of a cw-Ar ${ }^{+}$laser, with a power density between 1.0 and $10.0 \mathrm{~kW} \mathrm{~cm}^{-2}$, exciting up to five excitons/CQD.

Figure 2(a) depicts the evolution of the $\mu$-PL of a single $\mathrm{CQD}$, with the increase of the power density (when $P_{0}=1.0 \mathrm{~kW} \mathrm{~cm}^{-2}$ ). The spectra in figure 2(a) consist of multiple bands, each demonstrates independent behavior on the increase of the laser power. A contour plot of a $\mu$-PL time evolution is shown in figure 2(b). This plot depicts important characteristics; the intensity of the dominant bands (expressed by the blue regions in the contour) undergoes only minor intensity fluctuations of $<10 \%$ (see side ruler) and a small spectral diffusion, suggesting $\sim 90 \%$ blinking-free behavior. Furthermore, a single $\mathrm{CdTe} / \mathrm{CdTe}_{x} \mathrm{Se}_{1-x} \mathrm{CQD}$ showed extended robustness throughout a few weeks of measurements. This is in contrast to the nature of a single core CdTe, showing only a single band spectrum, which in most cases, deteriorates after a trace of a single $\mu$-PL frame and cannot be investigated properly. The assignment of the multi-bands in figure 2(a) are discussed, referring to the lowest electronic states $\left(1 \mathrm{~S}_{\mathrm{h}}, 1 \mathrm{P}_{\mathrm{h}}, 1 \mathrm{~S}_{\mathrm{e}}, 1 \mathrm{P}_{\mathrm{e}}\right)$, which are drawn schematically as a cascade of diagrams in the insets in panel (a), with the electrons and hole marked by the filled dots and the open dots, respectively. The red band showed an initial linear increase of the emission intensity up to $\sim 3.5 P_{0}$, which can be attributed to a single-exciton $(X \rightarrow 0)$ recombination, as sketched in the diagram (often called ground state exciton with a label $X^{0}$ ). The green band showed a quadratic increase up to $\sim 6.5 P_{0}$, with increased laser power, marking it as the ground state biexciton $(2 X \rightarrow X)$ recombination. Above the indicated thresholds, other broader bands (blue and yellow) emerged simultaneously on the red side and on the blue side of the 


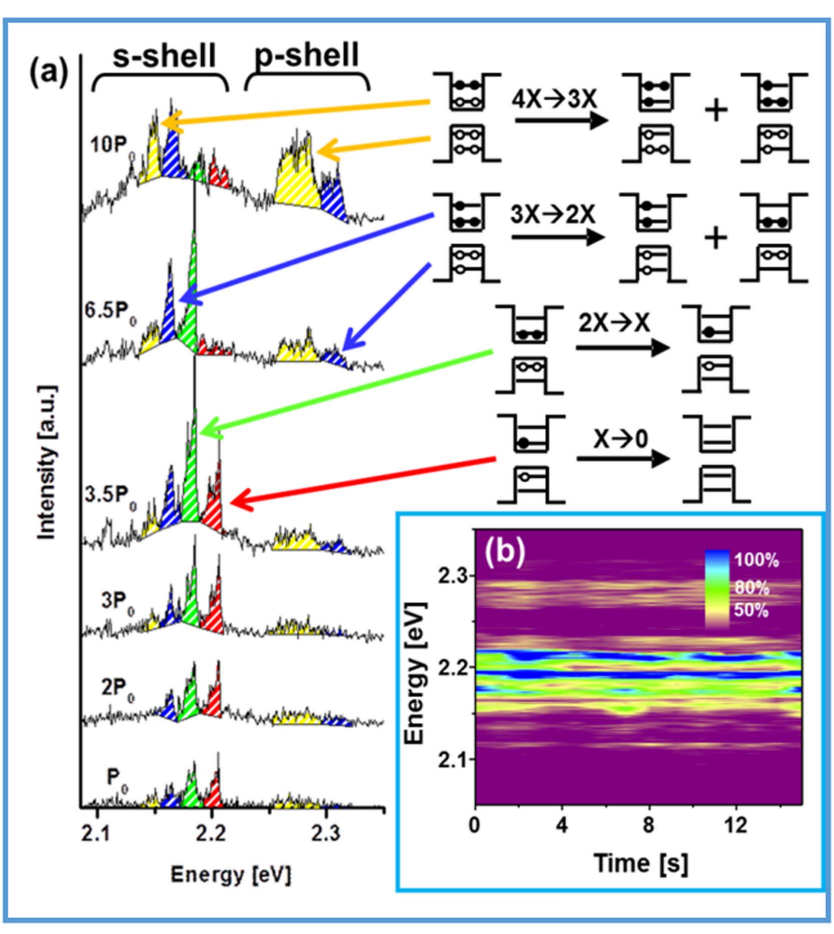

Figure 2. (a) Representative evolution of $\mu$-PL spectra of an individual $\mathrm{CdTe} / \mathrm{CdTe}_{x} \mathrm{Se}_{1-x}$ core/alloyed-shell CQD as a function of the excitation power when $P_{0}=1 \mathrm{~kW} \mathrm{~cm}^{-2}$. The spectra consist of 4-6 bands (shaded in different colors) that adapt independently to the intensification of the laser power, associated with the formation of single- $(X)$, bi- $(2 X)$, tri- $(3 X)$ and four-excitons $(4 X)$. The excited and ground electronic states of the relevant transitions are drawn schematically as a cascade of diagrams. The recombination routes are drawn schematically in the diagram. (b) A contour plot, presenting the variation of the $\mu$-PL intensity versus time and energy, revealing a blinking-free behavior.

spectra, and the $X$ and $2 X$ lines almost fade away. It is also important to note that each band is accompanied with weak sidebands that might be related to acoustic phonons ( $\sim 4 \mathrm{meV}$ ) or to an accidental occurrence of charged excitons, such as $X^{+}, 2 X^{+}$. The $2 X$ binding energy, related to the energy difference between the $X$ and $2 X$ bands $\left(\Delta E=E_{X}-E_{2 X}=23.4 \mathrm{meV}\right)$ is in close agreement to a calculated value (as reported in [130]), as well as close to a value found in self-assembled CdTe quantum dots of similar size [131]. The value of $\Delta E>0$ reflects an attractive $X-X$ Coulomb interaction in the $\mathrm{CdTe} / \mathrm{CdTe}_{x} \mathrm{Se}_{1-x}$ CQDs investigated. The blue and yellow bands in figure 2(a) developed at power densities $>6 P_{0}$, assigned to the $3 X \rightarrow 2 X$ and $4 X \rightarrow 3 X$ recombination, appearing on the red side of the spectrum with binding energies comparable with $E_{4 X}-E_{3 X}$ $\sim 17.5 \mathrm{meV}$ and $E_{3 X}-E_{2 X} \sim 18.6 \mathrm{meV}$; (b) $p$ shell recombination, appearing on the blue side of the spectrum and shifted by $70-100 \mathrm{meV}$ above the $\mathrm{X}$ band. The last shift is smaller than the energy spacing between the $\boldsymbol{p}$ and $\boldsymbol{s}$ shells in a zinc-blende CdTe CQD of similar size, due to the additional carriers' Coulomb interactions. The generation of steady multiple excitons has been a topic of interest during the past decade, due to their anticipated potential to increase photovoltaic efficiency above the Shockley-Queisser limit [132].
The current study indicates that specialized CQD design can sustain multiple excitons (from 0.1 to a few nanoseconds) under continuous-wave excitation for charge extraction in photovoltaic and catalysis applications.

\section{CdTe/CdSe giant core/shell CQDs}

The sample investigated consisted of CdTe cores of nearly spherical and tetrahedral shape, passivated with a two monolayer thickness of CdSe shell. The synthesis of CdTe/ $\mathrm{CdSe}$ core/shell CQDs is described in [133]. The overall core/shell CQDs had a diameter $(D)$ ranging from 8.5 to $25.5 \mathrm{~nm}$, below or above the bulk Bohr exciton radius $(9 \mathrm{~nm})$, featuring a (strong) moderate to weak or even marginal degree of quantum confinement. The magneto-optical properties were examined by following the $\mu$-PL spectrum of individual CQDs at cryogenic temperatures under a variable continuous-wave excitation power and in the presence of a magnetic field (up to 5 Tesla), combined with detection of the circular or linear emission components. The $\mu$-PL spectra of single CQDs were recorded using a fiber-based confocal microscope, immersed in a cryogenic system. The CQDs were dispersed over a silicon substrate with a density $<1 \mathrm{CQD} \mu \mathrm{m}^{-2}$ and were excited by an unpolarized continuous wave diode laser $\left(E_{\mathrm{exc}}=1.87 \mathrm{eV}\right)$ with diffraction limited spot diameter of $\sim 0.7 \mu \mathrm{m}$ and a spectral resolution of $300 \mu \mathrm{eV}$. The variable magnetic field was applied in Faraday configuration, normal to the substrate. The polarizationsensitive detection of the linear and circular components of the exciton emission were recorded by achromatic quarterwave plate and linear polarizer.

Representative $\mu$-PL spectra of an individual CQD with a diameter of $12.5 \mathrm{~nm}$, excited by a variable laser power in the absence of a magnetic field, are depicted in figure 3(a). The evolution of $\mu$-PL spectra with the increase of the excitation power from $P_{0}=25 \mathrm{~W} \mathrm{~cm}^{-2}$ to $P_{\max }=1.5 \mathrm{~kW} \mathrm{~cm}^{-2}$ reveals a distinction of three bands, marked in blue, red and green lines. The estimated average number of generated excitons $\langle N\rangle$ (see [133]), which vary from one to six, are labeled on the figure. The blue band grows linearly, while the other bands increase superlinearly with respect to the increase of the laser power, suggesting the association of the blue band to a single ground state exciton $(X)$, the red band to a bi-exciton $(2 X)$ and the green bands to ground state and excited state tri-excitons (positioned below or above the exciton band, respectively). Figure 3(b) displays a time trace of the exciton band intensity, revealing blinking-free behavior. Theoretical calculations (published in [133]) of the electronic band structure with an additional perturbative Coulomb interaction revealed the binding energy of the bi-exciton to be $\sim 3 \mathrm{meV}$ for a CQD of $12.5 \mathrm{~nm}$, which is in close agreement with the energy gap between the $X$ and $2 X$ bands shown in figure 3(a).

Characteristic linear and circular polarized $\mu$-PL spectra of an individual CdTe/CdSe CQD with a diameter of $9.5 \mathrm{~nm}$, recorded with/without the influence of an external magnetic field, are shown in figure 4(a). The top panel shows unpolarized $\mu$-PL spectrum of a CdTe/CdSe giant core/shell. The spectrum was recorded under excitation power that generated 


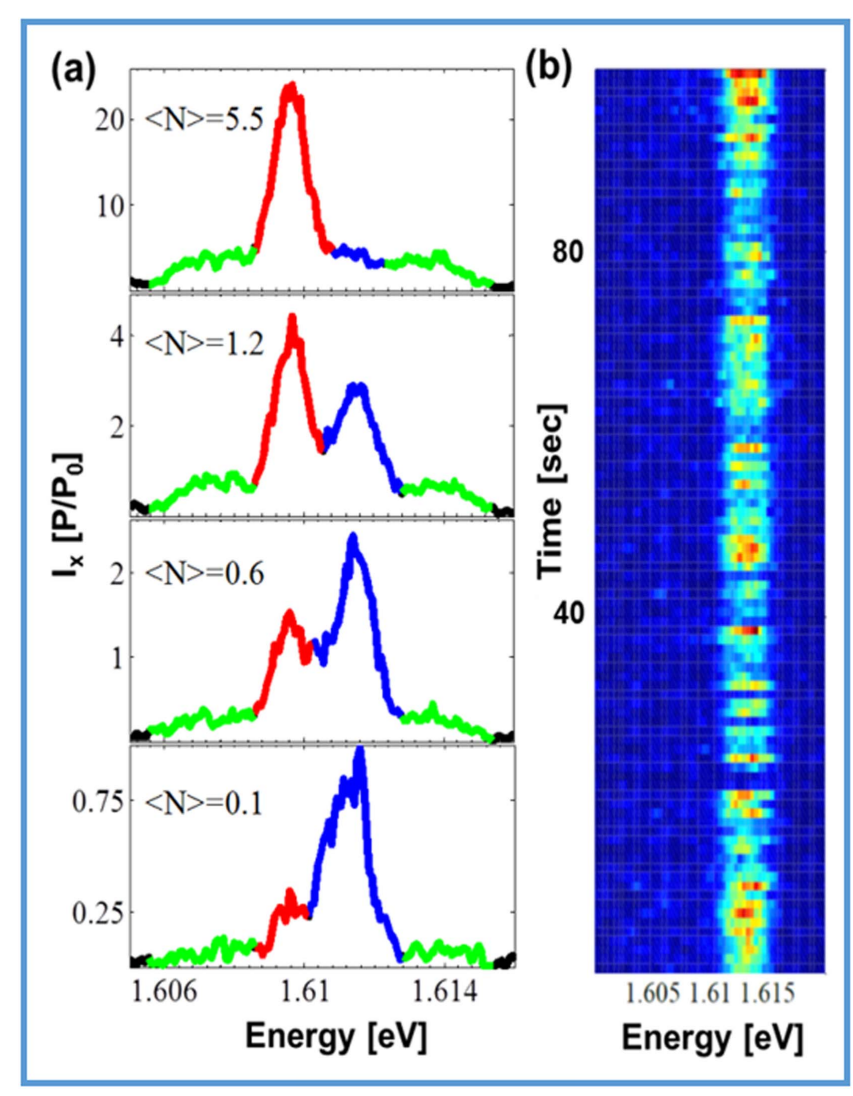

Figure 3. Evolution of $\mu$-PL spectra of a single $12.5 \mathrm{~nm} \mathrm{CdTe} / \mathrm{CdSe}$ giant core/shell CQD with an increase of the laser power from $P_{0}=25 \mathrm{~W} \mathrm{~cm}^{-2}$ to $P_{\max }=1.5 \mathrm{~kW} \mathrm{~cm}^{-2}$ (from bottom to top). The estimated average number of excitons $\langle N\rangle$ is indicated. The blue, red and green colors correspond to the exciton, bi-exciton and tri-exciton spectral regimes. (b) Time-trace of the exciton emission, revealing steady luminescence emission (blinking free behavior).

a single and biexciton at $4.2 \mathrm{~K}$. The corresponding polarized spectra are shown in the panels below. The bottom panel represents the linearly polarized emission components $\left(\Pi_{x}\right.$ and $\left.\Pi_{y}\right)$ when recorded at $B_{0}=0$. The middle and upper panels represent circular polarized components $\left(\sigma^{+}\right.$and $\left.\sigma^{-}\right)$ when recorded at $B_{0}=0$ and at $B_{0}=2 \mathrm{~T}$, respectively. Interestingly, the linearly and circularly polarized spectra recorded at similar $B_{0}$ show a small relative energy shift, but they have a comparable characteristic-an energy gap $\leqslant 1 \mathrm{meV}$ occurs between components, which grows with the increase of the $B_{0}$ strength. The existence of distinctive polarized components for excitons and bi-excitons suggests the occurrence of a slight shape distortion from a perfect cubic structure into prolate or oblate shapes with a semi-major axis. Indeed, the energy gap between the polarized components deviated from one CQD to another of the same size, presumably due to a random orientation of their semi-major axis on the sample holder, with respect to the observation direction. The polarization properties of the excitons are associated with electronic fine structure, created by a combination of effects, including the crystal field, electron-hole exchange interaction and shape distortion, as displayed in figure 5 for a ground state single exciton $(X)$ [26]. The co- existence of linear and circular polarization result from close proximity of the $|+1\rangle$ and $|-1\rangle$ states $\left(360 \mu \mathrm{eV}\right.$ at $\left.B_{0}=0\right)$ [133], and in combination with a shape distortion, allows mixing such as $|+1\rangle \pm \mathrm{i}|-1\rangle$, which generates the linear polarization components, $\Pi_{x}$ and $\Pi_{y}$. It should be noted that a ground state bi-exciton $(2 X)$ consists of dark $(J= \pm 2)$ and bright $(J=0)$ states; the latter is marked in figure 5 , referring to the $2 X \rightarrow X$ transition as recorded at $4 \mathrm{~K}$. The $2 X$ emission band shows cross polarization (see figure 4 (a)) with respect to the polarization of $X$. Figure 4(b) shows plots of peak energies shift $\left(E_{\sigma}\left(B_{0}\right)-E_{\sigma}\left(B_{0}=0 \mathrm{~T}\right)\right)$ of the exciton $(X)$ polarized components versus strength of $B_{0}$ for CQDs with $D=8.5 \mathrm{~nm}$ (top panel) and for CQDs with $D=25.5 \mathrm{~nm}$ (bottom panel); the former displays a linear dependence, while the latter presents a quadratic dependence on $B_{0}$. The curves were fitted to an equation read as $E_{\sigma \pm}\left(B_{0}\right)=E_{\sigma \pm}(0) \pm$ $\frac{1}{2} g_{X} \mu_{B} B+\gamma_{2} B^{2}$, where $E_{\sigma}\left(B_{0}\right)$ corresponds to the polarized component peak energy at field strength $B_{0}$, the second term is related to the energy shift described by a Zeeman interaction when $g_{x}$ is the exciton Landé $g$-factor, while the third term corresponds to a diamagnetic energy shift with a coefficient $\gamma_{2}$. Furthermore, $\gamma_{2}$ is related to the average exciton localization region $\left(r_{x}\right)$ via the relation $\gamma_{2}=e^{2}\left\langle r_{X}^{2}\right\rangle / 8 \mu$; $e$ is electron charge, $\mu$ - is electron hole reduced mass. Thus, the effective exciton diameter $\left(D_{X}\right)$ is according to the relation $D_{X}=2\left\langle r_{X}^{2}\right\rangle^{1 / 2}$. Figure 4(c) shows plots of the extracted $g_{\mathrm{x}}$ and $\gamma_{2}$ values, versus the diameter of the CQDs $(D)$ or versus the ratio $D x / D$. The plot reveals pronounced changes in $g_{x}$ and $\gamma_{2}$ with the increase of the CQD size: the exciton Landé $g$-factor value decreases by an order of magnitude with the increase of the CQD's diameter to $25.5 \mathrm{~nm}$, finally approaching the bulk limit of 0.2 ; the diamagnetic energy shift value increases with the increase of the size nearly up to the bulk limit of $14.7 \mu \mathrm{eV} \mathrm{T}^{-2}$ (bulk values are marked on the panel). The diamagnetic shift constant provides the information about effects of confinement and the Coulomb interaction in CQDs. In particular, for the $25.5 \mathrm{~nm}$ CQDs, the nearly bulk value of $D x$ reflects that Coulomb interactions confine the $X$ and $2 X$ excitons to a volume characterized by the Bohr radius, substantially smaller than the actual size of the CQDs. It is most likely that the excitons in the giant core/shell CQDs are focused at the central part of the dot, maintaining a uniform dielectric surrounding, resembling self-assembled quantum dots within a semiconductor host, and approaching excitons' behavior in bulk semiconductors. Thus, the influence of the exterior surfaces and the low dielectric constant of the organic ligands are substantially reduced, avoiding carriers' dielectric confinement and surface trapping. Screening the exciton from the exterior surrounding increases the spectral stability of the exciton emission, enabling the exploration of exciton properties in a platform nearly defect-free, with spectral resolution that cannot be achieved in bulk semiconductors. From an application viewpoint, giant-core/shell CQDs of the type discussed could be useful as light sources or spin-based devices. 


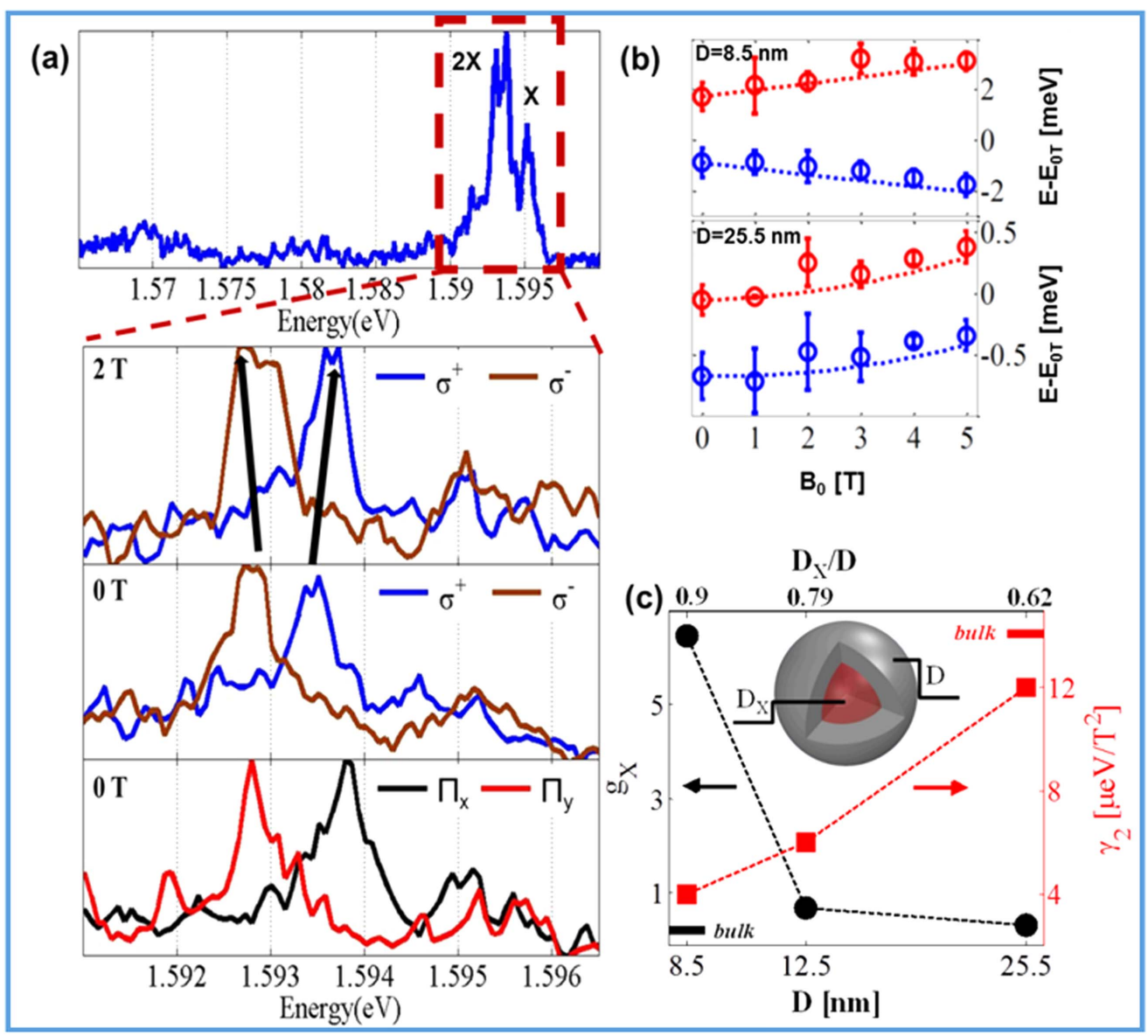

Figure 4. (a) Representative $\mu$-PL spectrum of a single $9.5 \mathrm{~nm} \mathrm{CdTe} / \mathrm{CdSe}$ giant-core/shell CQD (top) and the relevant linear ( $\Pi_{x}$, $\Pi_{y}$ ) (bottom) and circular $\left(\sigma^{+}, \sigma^{-}\right)$polarized spectra (middle), recorded at the indicated magnetic field strengths, $B_{0}$. (b) Plots of the polarized exciton components peak energy shift at $B_{0} \neq 0$ (away from its energy at $B_{0}=0$ ) versus strength of $B_{0}$, of two different size CQDs with diameter $(D)$ as indicated on the panel. (c) Plot of the extracted values of $g_{\text {ex }}$ and $\mathrm{\gamma}_{2}$, versus diameter $(D)$ or exciton-to-CQD diameter ratio $\left(D_{x} / D\right)$. The bulk values are marked on the panel.

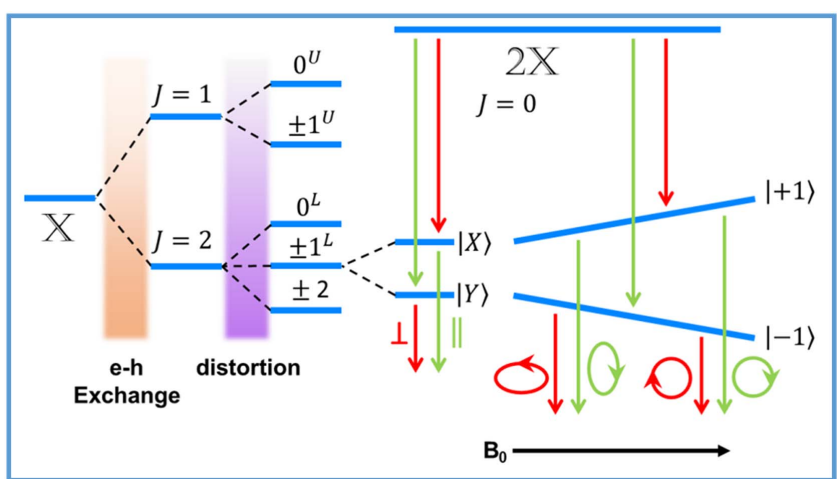

Figure 5. Schematic plot showing in details an exciton $(X)$ fine structure, induced by the following interactions: electron-hole exchange; crystal field or shape distortion; Zeeman interaction with external magnetic field $B_{0}$. The last split the bright state into circular polarized $\left(\sigma+, \sigma^{-}\right)$components, at the highest fields but, mixer of the states toward linear polarization $\left(\Pi_{x}, \Pi_{y}\right)$ components at lower field or/and elliptical polarization in the mid-regime of magnetic field. The lowest level of a biexciton $(2 X), J=0$, and the possible crossed polarized transitions $2 X \rightarrow X$ are marked on the diagram.

\section{Mn-doped CdTe/CdSe CQDs}

We review here the sp-d exchange interaction in $\mathrm{CdTe} / \mathrm{CdSe}$ core/shell CQDs embedded with low dilution of $\mathrm{Mn}^{+2}$ ions, incorporated selectively either in the core or in the shell. The synthesis of Mn-doped CdTe/CdSe core/shell CQDs is described in [134]. The studied CQDs were dispersed in 2,2,4,4,6,8,8-heptamethylnonane to form a glassy solution, and mounted into cryogenic system. The samples were pumped by an unpolarized continuous-wave $514.5 \mathrm{~nm}$ line of a cw- $\mathrm{Ar}^{+}$laser, at $2.2 \mathrm{~K}$. The circular polarized emissions were detected by a quarter-wave plate and linear polarizer combination. The ground-state electron configuration of a $\mathrm{Mn}^{+2}$ ion $\left({ }^{6} \mathrm{~A}_{1}\right)$ consists of five unpaired spins occupying the 3d-atomic orbitals, with total spin momentum $S_{\mathrm{Mn}}=5 / 2$. The first excited state $\left({ }^{4} \mathrm{~T}_{1}\right)$ is composed of three unpaired spins with $S_{\mathrm{Mn}}=3 / 2$. In the present case, the host lattice energy band gap is nearly in resonance with the ${ }^{4} \mathrm{~T}_{1}{ }^{6} \mathrm{~A}_{1}$ gap, a suitable condition for an efficient sp-d exchange interaction.

Figures $6(\mathrm{a})$ and (b) displays circularly polarized $\mu$-PL spectra (recorded at $4 \mathrm{~K}$ ) of an ensemble of $\mathrm{CdTe} / \mathrm{CdSe}$ CQDs, embedded with $\mathrm{Mn}^{+2}$ ions either in the core (a) or in the shell (b). A detailed description of the magnetic ion 

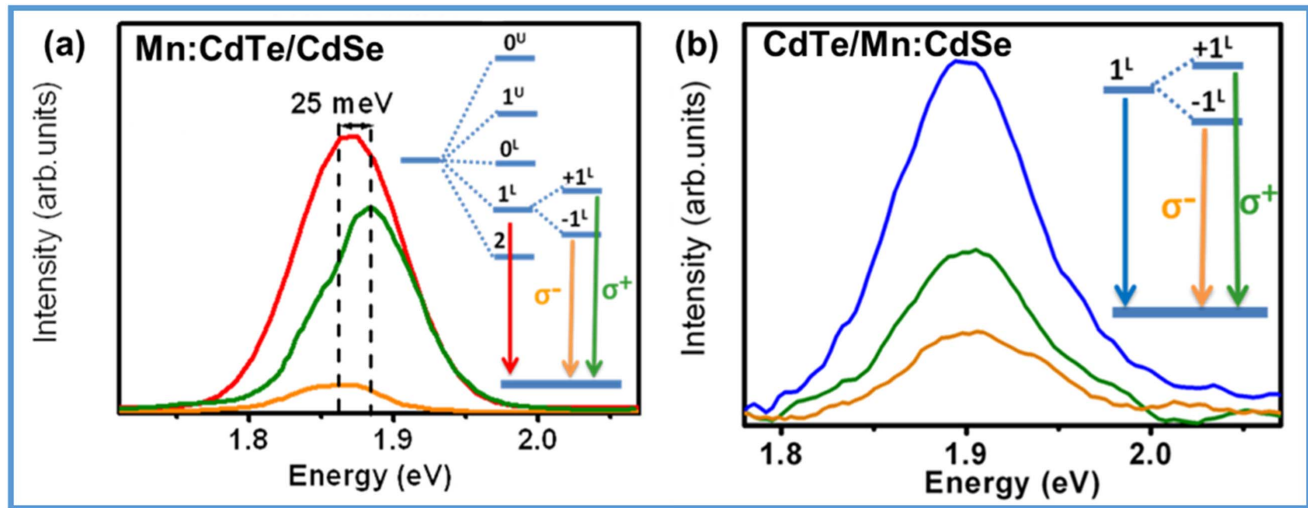

Figure 6. Polarized emission spectra of ensemble of CdTe/CdSe CQDs embedded with $\mathrm{Mn}^{+2}$ ions at the core (a) and at the shell (b), designating a larger effect for the hole- $\mathrm{Mn}^{+2}$ interaction, as anticipated theoretically (see text).

positioning is given in [135]. It is important to recall at this point that $\mathrm{CdTe} / \mathrm{CdSe} \mathrm{CQDs}$ possess quasi-type-II configuration, enabling exploration of the individual hole- $\mathrm{Mn}^{+2}$ and electron- $\mathrm{Mn}^{+2}$ interactions, when guest ions are positioned at different radial distances. The spectra in panel (a) show a pronounced energy gap $(\sim 20 \mathrm{meV})$ between the circular polarized components, in contradiction to the small gap $(\sim 3 \mathrm{meV})$ depicted in panel (b). Generally, the interaction energy of a carrier with magnetic ions is given by the relation: $\Delta E=\left\langle S_{z}\right\rangle N_{0}\left(f_{\mathrm{e}} \alpha-f_{\mathrm{h}} \beta\right)$, where $\left\langle\mathrm{S}_{z}\right\rangle$ is an effective spin moment of the $\mathrm{Mn}^{2+}$ being $5 / 2$ at $4 \mathrm{~K}, N_{0}$ is the density of the host lattice, $\alpha$ arises from potential ferromagnetic $\mathrm{s}-\mathrm{d}$ exchange and $\beta$ derives from kinetic-type antiferromagnetic $\mathrm{p}-\mathrm{d}$ exchange, $f_{\mathrm{e}}$ and $f_{\mathrm{h}}$ are characterized as the degree of spatial overlap between the $\mathrm{Mn}^{2+}$ wave function with the distribution functions of the electron and hole, respectively, and can be roughly approximated as the percentage of the carrier wave function within the core or within the shell. Assuming an isolation of the $\mathrm{p}-\mathrm{d}$ and $\mathrm{s}-\mathrm{d}$ interactions in the present case, the following values: $N_{0} . \alpha=0.009 \mathrm{eV}$ and $N_{0} . \sim 0.081 \mathrm{eV}$ have been extracted from the experimental data. A dominancy of the hole- $\mathrm{Mn}^{+2}$ has been commonly found before $[135,136]$, which may be due to the symmetry restricted s-d hybridization [136]. Also worth noting, the hole- $\mathrm{Mn}^{+2}$ interaction renders a degree of polarization larger than the pristine $\mathrm{CdTe} / \mathrm{CdSe}$ of $\sim 1 \mathrm{meV}$ at $B_{0}=0$ (see example in figure 4(b)), hence, magnetic doping induces dramatic changes of the exciton polarization, beneficial in designing spintronic devices.

\section{Halide perovskites CQDs}

In this section, we review the interplay of Rashba spin-orbit coupling on the band-edge exciton of $\mathrm{CsPbBr}_{3}$ CQDs [137]. Optical spectroscopy was performed on single dots with $\sim 10 \mathrm{~nm}$ diameter, measuring the linearly and circularly polarized single-photon emission under an applied magnetic field at $4 \mathrm{~K}$. Monodispersed $\mathrm{CsPbBr}_{3} \mathrm{CQDS}$ were prepared by solution-phase synthesis described in [138]. The $\mathrm{CsPbr}_{3}$ CQDs were spread onto a silicon substrate with density $<8.3 \mu \mathrm{g} \mathrm{cm}^{-3}$. The samples were excited by an unpolarized continuous wave diode laser $\left(E_{\mathrm{exc}}=2.75 \mathrm{eV}\right)$ with diffraction limited spot diameter of $\sim 0.5 \mu \mathrm{m}$ and power fluency $<50 \mathrm{~W} \mathrm{~cm}^{-2}$. The variable magnetic field was applied in Faraday configuration, normal to the substrate. The polarization-sensitive detection of the linear and circular components of the exciton emission were recorded by achromatic quarter-wave plate and linear polarizer, with spectral resolution of $400 \mu \mathrm{eV}$. Figure 7(a) shows unpolarized $\mu$-PL spectra of two different $\mathrm{CsPbBr}_{3} \mathrm{CQDs}$, recorded with and without a magnetic field $\left(B_{0}\right)$ as labeled in the panels. The spectra show a blinking-free behavior, which might be due to the relatively large size of the dots compared with the ground state exciton Bohr radius of $\sim 4.5 \mathrm{~nm}$ [139], or due to a low occurrence of inter-band defect states [140-142]. The spectra at $B_{0}=0 \mathrm{~T}$ in figures 7(a), (b) include a dominant low-energy band associated with the band-edge exciton recombination, labeled as regime I, accompanied by two peaks blue-shifted by $2.3 \mathrm{meV}$ (labeled as regime II) and by $5.7 \mathrm{meV}$ (labeled as regime III). In addition, a few weak side bands appear on both sides. The band I was best fitted to two Lorentzian functions (not shown here), revealing an energy split of $0.16-0.4 \mathrm{meV}$ at $0 \mathrm{~T}$ and a pronounced split of $1.2 \mathrm{meV}$ at $8 \mathrm{~T}$. Figure 7(b) displays a plot comprised of collection of points referring to the split components' energies of the excitons from 1500 different scans, versus strength of the applied magnetic field $B_{0}$. Figure 7(c) displays a plot of the energy gap between the exciton split component $(\Delta E)$ versus the strength of $B_{0}$ (red squares). The dependence of $\Delta E$ on $B_{0}$ cannot be fit to a linear Zeeman function (green curve) nor to a quadratic Zeeman function (blue curve) [143, 144]. However, the results can be qualitatively understood by a combined Rashba and Zeeman model as discussed below (see black circles in the figure). The origin of emission bands in regimes II and III is currently uncertain, but might be related to anti-Stokes acoustic phonons or high order excitons.

Figure 8 presents sets of polarized $\mu$-PL spectra of a single $\mathrm{CsPbBr}_{3} \mathrm{CQD}$. The emission bands in figure 8(a) were detected with a linear polarizer, rotated between two orthogonal orientations (red and blue curves) with respect to a laboratory axis. The blue and red curves in panel (a) have peaks at different locations. This is most clearly displayed at a specific orthogonal position of $60^{\circ} / 150^{\circ}$ (middle spectra), 

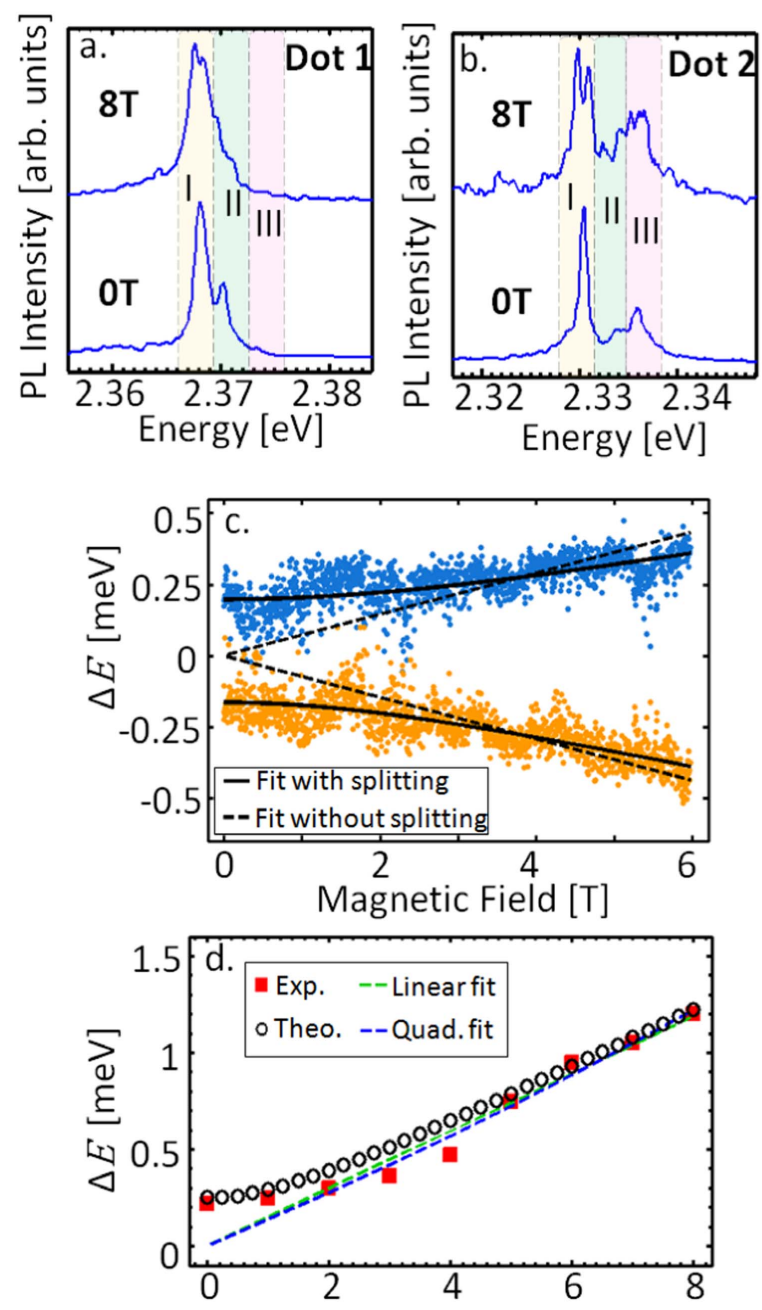

Figure 7. (a), (b) Representative $\mu$-PL spectra of a single $\mathrm{CsPbBr}_{3}$, recorded at different $B_{0}$ strengths. The spectra consists of three regimes, when the low energy at regime I is associated with a single exciton; (c) plot of the exciton split component positions (away from a central point) versus $B_{0}$ from 1500 different scans (black lines: attempts for a fit); (d) plot the average split energy $(\Delta E)$ between split components versus strength of $B_{0}$ (red symbols). Other lines in the panel refer to different fitting procedures, as indicated in the legend. Theoretical model included consideration of Rashba and Zeeman effects as described in the text.

probably due to coincident alignment of one detection angle with the unique crystallographic axis. The emission bands in figure 8(b) were excited by unpolarized light, but detected via left and right circular polarizers. Similar to the linearly polarized spectra, the spectra show splitting of the exciton band with increased strength of $B_{0}$. The coexistence of both linear and circular polarization of the same optical transitions, as shown in figure 8, indicates the presence of elliptical polarization.

As mentioned, the $\left[\mathrm{PbBr}_{6}\right]^{-4}$ network dominates the electronic band structure. However, the $A$-site ions have special impact on the physical properties, due to their vibrational degrees of freedom. It has been previously proposed $[145,146]$ that polar organic ions (e.g., MA or FA) form hydrogen bonds to the halide anions, which induce significant structural changes via displacement of the $X$ and $M$ ions [147, 148]. Similarly, displacement freedom of a small inorganic ion like $\mathrm{Cs}^{+}$within the cuboctahedral void might induce a net crystalline distortion [149], which could lead to (local or global) inversion-symmetry breaking and/or a buildup of a local electric field [145-147, 149-158]. Then, in the frame of reference of a moving electron, an electric field transforms into a magnetic field, coupling electron spin with its momentum. This effective magnetic field lifts degeneracy of the spin-degenerate bands, a phenomenon known as Rashba effect [119, 159-162]. The lift of degeneracy may be related also to a Dresselhaus effect [163]. However, recent theoretical studies indicated a major contribution from the Rashba effect in perovskites materials. In particular, symmetry considerations predicted a pure Rashba band structure for lead halide perovskites, such as $\mathrm{CsPbBr}_{3}$, whereas other Sn-based halides could have mixed Rashba and Dresselhaus splitting [164]. The perovskites under investigation have a low symmetry of an orthorhombic structure, as well dominant heterogeneity. Indeed, recent theoretical investigations $[119,159,160]$ predicted dominancy of the Rashba effect in lead halide perovskites, hence, laid a ground for the assumptions made in this work. Figure 8(c) provides an illustration of the absorption and emission processes occurring between Rashba split band-edges in the zero magnetic field limit, when the emission process involves a quasi-forbidden recombination. The Rashba effect modifies the exciton electronic structure, inducing a zero magnetic field splitting of the spin-degenerate excitons. The magnitude of this splitting is set by the size of the spin-orbit interaction, and the amount of centrosymmetry breaking induced near the nanocrystal surfaces. The most recent effort (published elsewhere) featured the development of a model including both the Rashba and Zeeman effect and supplied the strongest support for the experimental evidence shown in figures 7 and 8. Taking into account the Rashba and magnetic-field effects, the Hamiltonian for the exciton relative coordinate (r) degree of freedom is given by: $H=-\frac{\vec{\nabla}_{\mathrm{r}}^{2}}{2 m_{\mathrm{r}}}+V(r)+$ $\left(\alpha_{\mathrm{e}} \vec{\sigma}_{\mathrm{e}}-\alpha_{\mathrm{h}} \vec{\sigma}_{\mathrm{h}}\right) \cdot\left(\hat{n} \times \mathrm{i} \vec{\nabla}_{\mathrm{r}}\right)+\frac{1}{2} \mu_{B} \vec{B} \cdot\left(g_{\mathrm{e}} \vec{\sigma}_{\mathrm{e}}-g_{\mathrm{h}} \vec{\sigma}_{\mathrm{h}}\right)$. Where, $V(r)$ is the electron-hole interaction, $m_{\mathrm{r}}$ is the reduced mass, $\alpha_{\mathrm{e}}$ and $\alpha_{\mathrm{h}}$ are Rashba coefficients for electron and hole, and $g_{\mathrm{e}}$ and $g_{\mathrm{h}}$ are $g$-factors for electron and hole. The spin degrees of freedom in the conduction-band manifold $\left(m_{J}= \pm 1 / 2\right)$ and valence-band manifold $\left(m_{S}= \pm 1 / 2\right)$ states are represented by the Pauli matrices, $\vec{\sigma}_{\mathrm{e}}$ and $\vec{\sigma}_{\mathrm{h}}$, respectively. The model indicated that as the magnetic field is increased, the magnitude of the Zeeman splitting eventually dominates the magnitude of the Rashba-induced splitting. Beyond this point, the splitting of the exciton peaks increases linearly with the magnetic field. This explains the nonlinear evolution of peak splitting in figure $7(\mathrm{~d})$. The Rashba effect supports the character of perovskite materials with a long exciton diffusion length typical for a quasi-forbidden optical transition in the absence of non-radiative recombination. We note that this effect is seen in the lead-halide perovskite quantum dots but not in the II-VI dots because of the large spin-orbit coupling of the former. 


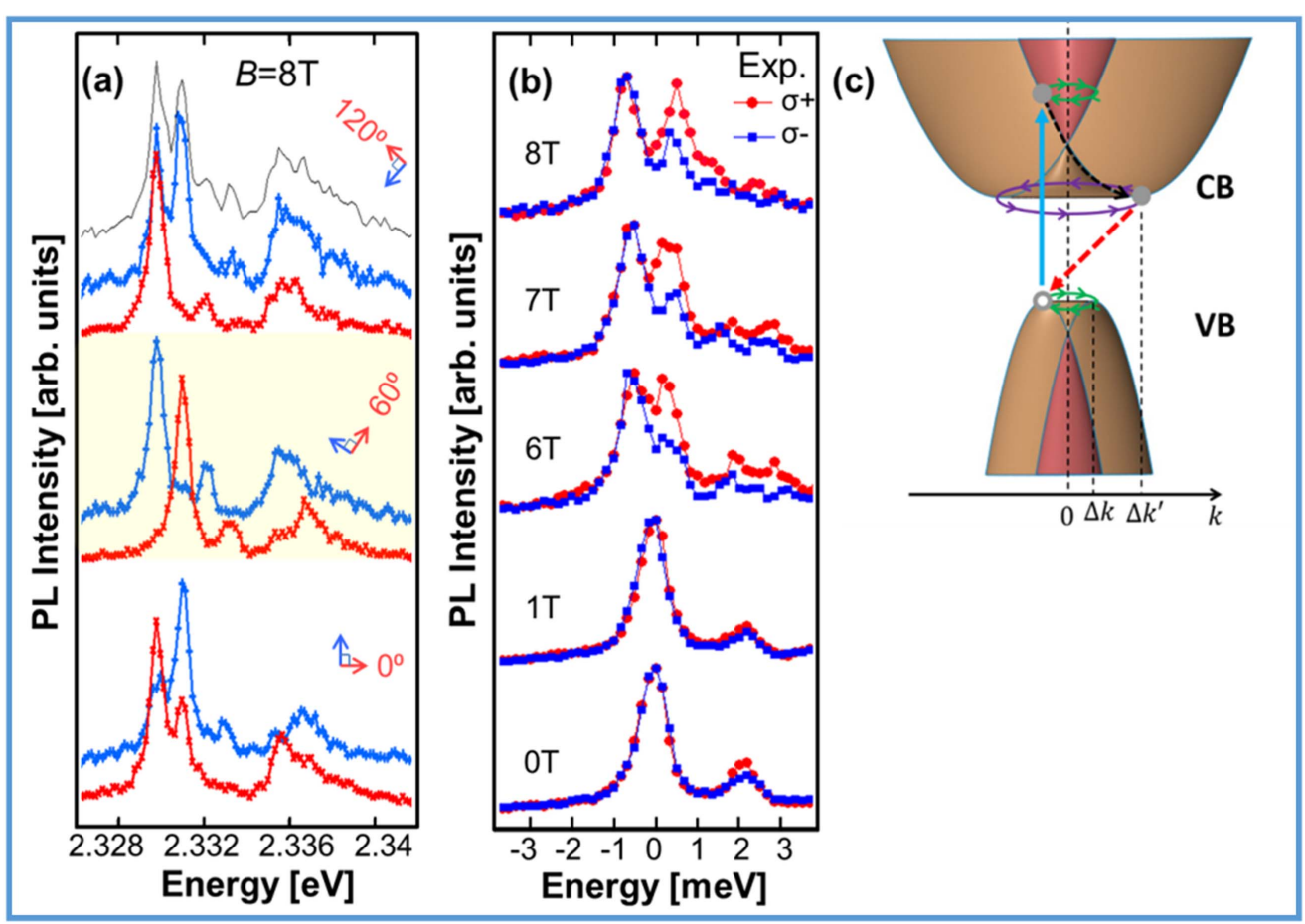

Figure 8. Set of $\mu$-PL spectra of a single $\mathrm{CsPbBr}{ }_{3} \mathrm{CQD}$, detected with linear polarizers (at two orthogonal directions) under magnetic field strength of $8 \mathrm{~T}$ (a) or detected with circular polarizers under various strengths of $B_{0}$ (b). Both the linear and circular polarized spectra exhibit split of the exciton lines (and the accompanied lines as well) in the absence of present of a magnetic field. A basic scheme, showing a Rashba effect is shown in the diagram in (c).

Besides the manifestation of Rashba effect in single excitons, lead-halide nanocrystals have also displayed physics related to charged exciton and biexciton formation $[165,166]$. These studies have revealed a rich exciton spectrum containing multiple sublevels, which were attributed to two different types of crystal structures present in the nanocrystals.

In summary, the polarized emission of CQD from II-VI and perovskite semiconductors were investigated thoroughly, revealing information of paramount scientific and technological interest about the optical transitions in semiconductor nanostructures. The studies included recording micro-photoluminescence of individual nanostructures at cryogenic temperatures, with or without the influence of an external magnetic field, and monitoring circular and/or linear polarized emission. The nanostructures investigated-core/ alloyed-shell and giant core/shell based on II-VI semiconductors, as well as the perovskite CQDs-showed blinking-free behavior in alloyed dots, due to a suppression of an Auger process; in giant dots due to reduction of the proximity to a surface; and in perovskites dots due to their size and higher level of tolerance to impurities. The blinking-free CQDs enabled detection of spectrally stable excitons and biexcitons under continuous-wave excitation. The binding energies were tuned by the size and architecture of the nanostructures. As indicated, biexciton binding energy was found to be $23 \mathrm{meV}$ in $\mathrm{CdTe} / \mathrm{CdTe}_{x} \mathrm{~S}_{1-x} \mathrm{CDQs}$ with a diameter of $\sim 4 \mathrm{~nm}$ and a quasi-type-II configuration, but only $3 \mathrm{meV}$ in $\mathrm{CdTe} / \mathrm{CdSe}$ CQDs with a diameter of $12.5 \mathrm{~nm}$ and type-I configuration.
Distinctive polarized transitions in II-VI CQDs originated from electron-hole exchange interaction combined with a shape distortion; though it is mainly dictated by the spinorbit Rashba effect in perovskite CQDs. Under the influence of an external static magnetic field, the polarized components had a Zeeman dependence in the II-VI CQDs under investigation, but deviated from a pure Zeeman effect in the perovskite CQDs due to contribution of a Rashba effect at field strengths $<4 \mathrm{~T}$.

Overall, the type and degree of polarization elucidated the exciton manifolds, angular momentum of the emitting states and Landé $g$-factors, with dependence on size, internal architecture and composition. In consequence, the study advanced the understanding of the materials' functionalities and elucidates the required design for various opto-electronic and spintronic devices.

\section{Acknowledgments}

The authors express their gratitude to the following scientists for their experimental complementary contribution: Dr Dima Cheskis, Dr Ruth Osovsky and Dr Georgy Maikov, for assembling of the experimental setup and for preliminary observation of single-dot research; Dr Nathan Grumbach and Dr Richard Capek for the synthesis of magnetically doped QDs. We express our thanks to Dr Youngjin Jang for assisting in the preparation of the manuscript. M I expresses her gratitude to the fellowship received by the Nancy and Stephen Grand Technion Energy Program. EL acknowledges 
the financial support from the Israel Council for High Education-Focal Area Technology (No. 872967), the Volkswagen Stiftung, (No.88116), the Israel Science Foundation, (No.914/15) and the Israel Science Foundation Bikura Program (No. 1508/14) and the European Commission via the Marie-Sklodowska Curie action Phonsi (H2020-MSCA-ITN642656). DV acknowledges the financial support from ERC Advanced Grant, nr. 692691-First Step; NWO-Physics, FOM program DDC 13 'Designing Dirac carriers in semiconductor superlattices'; NWO-CW, Toppunt 'Superficial superstructures'. LZT and AMR acknowledge support from the Office of Naval Research, under grant N00014-17-12574. MVK acknowledges financial support from the European Research Council under the European Union's Seventh Framework Program (Grant Agreement Nr. 306733, ERC Starting Grant 'NANOSOLID'). MIB is grateful to Swiss National Science Foundation (SNF Ambizione Energy grant, Grant Nr. PZENP2_154287).

\section{References}

[1] Talapin D V, Lee J-S, Kovalenko M V and Shevchenko E V 2010 Prospects of colloidal nanocrystals for electronic and optoelectronic applications Chem. Rev. 110 389-458

[2] Peng X, Manna L, Yang W, Wickham J, Scher E, Kadavanich A and Alivisatos A P 2000 Shape control of CdSe nanocrystals Nature 404 59-61

[3] Talapin D V, Rogach A L, Kornowski A, Haase M and Weller H 2001 Highly luminescent monodisperse CdSe and $\mathrm{CdSe} / \mathrm{ZnS}$ nanocrystals synthesized in a hexadecylaminetrioctylphosphine oxide-trioctylphospine mixture Nano Lett. 1 207-11

[4] Kim S, Fisher B, Eisler H-J and Bawendi M 2003 Type-II quantum dots: $\mathrm{CdTe} / \mathrm{CdSe}$ (core/shell) and $\mathrm{CdSe} / \mathrm{ZinTe}$ (core/shell) heterostructures J. Am. Chem. Soc. 125 11466-7

[5] Reiss P, Bleuse J and Pron A 2002 Highly luminescent CdSe/ $\mathrm{ZnSe}$ core/shell nanocrystals of low size dispersion Nano Lett. 2 781-4

[6] Brumer M, Kigel A, Amirav L, Sashchiuk A, Solomesch O, Tessler $\mathrm{N}$ and Lifshitz E $2005 \mathrm{PbSe} / \mathrm{PbS}$ and $\mathrm{PbSe} / \mathrm{PbSe}_{x} \mathrm{~S}_{1-X}$ core/shell nanocrystals Adv. Funct. Mater. 15 1111-6

[7] Chan E M, Alivisatos A P and Mathies R A 2005 Hightemperature microfluidic synthesis of CdSe nanocrystals in nanoliter droplets $J$. Am. Chem. Soc. 127 13854-61

[8] Kim T-H et al 2011 Full-colour quantum dot displays fabricated by transfer printing Nat. Photon. 5 176-82

[9] Erdem T and Demir H V 2016 Colloidal nanocrystals for quality lighting and displays: milestones and recent developments Nanophotonics 5 74-95

[10] Malko A V, Mikhailovsky A A, Petruska M A, Hollingsworth J A, Htoon H, Bawendi M G and Klimov V I 2002 From amplified spontaneous emission to microring lasing using nanocrystal quantum dot solids Appl. Phys. Lett. 81 1303-5

[11] Johnston K W, Pattantyus-Abraham A G, Clifford J P, Myrskog S H, Hoogland S, Shukla H, Klem E J D, Levina L and Sargent E H 2008 Efficient schottky-quantumdot photovoltaics: the roles of depletion, drift, and diffusion Appl. Phys. Lett. 92 122111-3

[12] Law M, Beard M C, Choi S, Luther J M, Hanna M C and Nozik A J 2008 Determining the internal quantum efficiency of PbSe nanocrystal solar cells with the aid of an optical model Nano Lett. 8 3904-10

[13] Etgar L, Yanover D, Capek R K, Vaxenburg R, Xue Z, Liu B, Nazeeruddin M K, Lifshitz E and Graetzel M 2013 Core/ Shell $\mathrm{PbSe} / \mathrm{PbS}$ QDs $\mathrm{TiO}_{2}$ heterojunction solar cell $A d v$. Funct. Mater. 23 2736-41

[14] Amirav L and Alivisatos A P 2010 Photocatalytic hydrogen production with tunable nanorod heterostructures $J$. Phys. Chem. Lett. 1 1051-4

[15] Amirav L and Alivisatos A P 2013 Luminescence studies of individual quantum dot photocatalysts $J$. Am. Chem. Soc. 135 13049-53

[16] Razgoniaeva N, Moroz P, Lambright S and Zamkov M 2015 Photocatalytic applications of colloidal heterostructured nanocrystals: what's next? J. Phys. Chem. Lett. 6 4352-9

[17] Kamat P V 2012 Boosting the efficiency of quantum dot sensitized solar cells through modulation of interfacial charge transfer Acc. Chem. Res. 45 1906-15

[18] Santra P K and Kamat P V 2012 Mn-doped quantum dot sensitized solar cells: a strategy to boost efficiency over $5 \%$ J. Am. Chem. Soc. 134 2508-11

[19] Bradshaw L R, Knowles K E, McDowall S and Gamelin D R 2015 Nanocrystals for luminescent solar concentrators Nano Lett. 15 1315-23

[20] Brumer M, Sirota M, Kigel A, Sashchiuk A, Galun E, Burshtein Z and Lifshitz E 2006 Nanocrystals of PbSe Core, $\mathrm{PbSe} / \mathrm{PbS}$, and $\mathrm{PbSe} / \mathrm{PbSe}_{x} \mathrm{~S}_{1-X}$ core/shell as saturable absorbers in passively $Q$-switched near-infrared lasers Appl. Opt. 45 7488-97

[21] Sukhovatkin V, Hinds S, Brzozowski L and Sargent E H 2009 Colloidal quantum-dot photodetectors exploiting multiexciton generation Science 324 1542-4

[22] Dubertret B 2005 Quantum dots: DNA detectives Nat. Mater. 4 797-8

[23] Smith A M and Nie S 2009 Next-generation quantum dots Nat. Biotechnol. 27 732-3

[24] Chappert C, Fert A and Van Dau F N 2007 The emergence of spin electronics in data storage Nat. Mater. 6 813-23

[25] Hirohata A and Takanashi K 2014 Future perspectives for spintronic devices J. Phys. D: Appl. Phys. 47193001

[26] Efros A L, Rosen M, Kuno M, Nirmal M, Norris D J and Bawendi M 1996 Band-edge exciton in quantum dots of semiconductors with a degenerate valence band: dark and bright exciton states Phys. Rev. B 544843

[27] Franceschetti A and Zunger A 2000 Optical transitions in charged CdSe quantum dots Phys. Rev. B 62 R16287

[28] Chamarro M, Gourdon C, Lavallard P, Lublinskaya O and Ekimov A I 1996 Enhancement of electron-hole exchange interaction in CdSe nanocrystals: a quantum confinement effect Phys. Rev. B 53 1336-42

[29] Piryatinski A, Ivanov S A, Tretiak S and Klimov V I 2007 Effect of quantum and dielectric confinement on the exciton -exciton interaction energy in type II core/shell semiconductor nanocrystals Nano Lett. 7 108-15

[30] Lifshitz E et al 2006 Air-stable $\mathrm{PbSe} / \mathrm{PbS}$ and $\mathrm{PbSe} / \mathrm{PbSe}_{X} \mathrm{~S}_{1-X}$ core-shell nanocrystal quantum dots and their applications $\dagger$ J. Phys. Chem. B 110 25356-65

[31] Roy Choudhury K, Sahoo Y, Jang S and Prasad P 2005 Efficient photosensitization and high optical gain in a novel quantum-dot-sensitized hybrid photorefractive nanocomposite at a telecommunications wavelength $A d v$. Funct. Mater. 15 751-6

[32] Klimov V I, Ivanov S A, Nanda J, Achermann M, Bezel I, McGuire J A and Piryatinski A 2007 Single-exciton optical gain in semiconductor nanocrystals Nature 447 441-6

[33] Caruge J M, Chan Y, Sundar V, Eisler H J and Bawendi M G 2004 Transient photoluminescence and simultaneous amplified spontaneous emission from multiexciton states in CdSe quantum dots Phys. Rev. B 7085316 
[34] Beard M C, Knutsen K P, Yu P, Luther J M, Song Q, Metzger W K, Ellingson R J and Nozik A J 2007 Multiple exciton generation in colloidal silicon nanocrystals Nano Lett. 7 2506-12

[35] Nair G and Bawendi M G 2007 Carrier multiplication yields of CdSe and CdTe nanocrystals by transient photoluminescence spectroscopy Phys. Rev. B 7681304

[36] McGuire J A, Joo J, Pietryga J M, Schaller R D and Klimov V I 2008 New aspects of carrier multiplication in semiconductor nanocrystals Acc. Chem. Res. 41 1810-9

[37] Trinh M T, Houtepen A J, Schins J M, Hanrath T, Piris J, Knulst W, Goossens A P L M and Siebbeles L D A 2008 In spite of recent doubts carrier multiplication does occur in PbSe nanocrystals Nano Lett. 8 1713-8

[38] Beard M C, Midgett A G, Hanna M C, Luther J M, Hughes B K and Nozik A J 2010 Comparing multiple exciton generation in quantum dots to impact ionization in bulk semiconductors: implications for enhancement of solar energy conversion Nano Lett. 10 3019-27

[39] Oron D, Kazes M and Banin U 2007 Multiexcitons in type-II colloidal semiconductor quantum dots Phys. Rev. B 75 35330

[40] Rabani E and Baer R 2010 Theory of multiexciton generation in semiconductor nanocrystals Chem. Phys. Lett. 496 227-35

[41] Hendry E, Koeberg M, Wang F, Zhang H, de Mello Donega C, Vanmaekelbergh D and Bonn M 2006 Direct observation of electron-to-hole energy transfer in CdSe quantum dots Phys. Rev. Lett. 9657408

[42] Jha P P and Guyot-Sionnest P 2007 Photoluminescence switching of charged quantum dot films J. Phys. Chem. C 111 15440-5

[43] Hyun B-R, Zhong Y-W, Bartnik A C, Sun L, Abruna H D, Wise F W, Goodreau J D, Matthews J R, Leslie T M and Borrelli N F 2008 Electron injection from colloidal PbS quantum dots into titanium dioxide nanoparticles ACS Nano 2 2206-12

[44] Kharchenko V A and Rosen M 1996 Auger relaxation processes in semiconductor nanocrystals and quantum wells J. Lumin. $70158-69$

[45] Efros A L and Rosen M 1997 Random telegraph signal in the photoluminescence intensity of a single quantum dot Phys. Rev. Lett. 781110

[46] Istvan R, Gresback R, Kortshagen U, Schaller R D and Klimov V I 2009 Universal size-dependent trend in auger recombination in direct-gap and indirect-gap semiconductor nanocrystals Phys. Rev. Lett. 102177404

[47] Jarosz M V, Porter V J, Fisher B R, Kastner M A and Bawendi M G 2004 Photoconductivity studies of treated CdSe quantum dot films exhibiting increased exciton ionization efficiency Phys. Rev. B 70195327

[48] Frantsuzov P, Kuno M, Jankó B, Marcus R A A, Janko B, Marcus R A A, Jankó B and Marcus R A A 2008 Universal emission intermittency in quantum dots, nanorods and nanowires Nat. Phys. 4 519-22

[49] Shimizu K T, Neuhauser R G, Leatherdale C A, Empedocles S A, Woo W K and Bawendi M G 2001 Blinking statistics in single semiconductor nanocrystal quantum dots Phys. Rev. B 63205316

[50] Dekel E, Regelman D V, Gershoni D, Ehrenfreund E, Schoenfeld W V and Petroff P M 2000 Cascade evolution and radiative recombination of quantum dot multiexcitons studied by time-resolved spectroscopy Phys. Rev. B 62 $11038-45$

[51] Efros A L and Rodina A V 1989 Confined excitons, trions and biexcitons in semiconductor microcrystals Solid State Commun. 72 645-9
[52] Rodina A V and Efros A L 2010 Band-edge biexciton in nanocrystals of semiconductors with a degenerate valence band Phys. Rev. B 82125324

[53] Schwartz O and Oron D 2012 A present understanding of colloidal quantum dot blinking Isr. J. Chem. 52 992-1001

[54] Tang J and Marcus R A 2005 Mechanisms of fluorescence blinking in semiconductor nanocrystal quantum dots J. Chem. Phys. 123054704

[55] Fernee M J, Plakhotnik T, Louyer Y, Littleton B N, Potzner C, Tamarat P, Mulvaney P and Lounis B 2012 Spontaneous spectral diffusion in CdSe quantum dots J. Phys. Chem. Lett. 3 1716-20

[56] Verberk R, van Oijen A M and Orrit M 2002 Simple model for the power-law blinking of single semiconductor nanocrystals Phys. Rev. B 66233202

[57] Jha P P and Guyot-Sionnest P 2010 Electrochemical switching of the photoluminescence of single quantum dots J. Phys. Chem. C 114 21138-41

[58] Nelson G A and Zhu X Y 2012 Reversible surface electronic traps in $\mathrm{PbS}$ quantum dot solids induced by an orderdisorder phase transition in capping molecules J. Am. Chem. Soc. $1347592-5$

[59] Asbury J B, Hao E, Wang Y, Ghosh H N and Lian T 2001 Ultrafast electron transfer dynamics from molecular adsorbates to semiconductor nanocrystalline thin films J. Phys. Chem. B 105 4545-57

[60] Maikov G I, Vaxenburg R, Sashchiuk A and Lifshitz E 2010 Composition-tunable optical properties of colloidal IV-VI quantum dots, composed of core/shell heterostructures with alloy components ACS Nano 4 6547-56

[61] Rabouw F T, Lunnemann P, van Dijk-Moes R J A, Frimmer M, Pietra F, Koenderink A F and Vanmaekelbergh D 2013 Reduced auger recombination in single $\mathrm{CdSe} / \mathrm{CdS}$ nanorods by one-dimensional electron delocalization Nano Lett. 13 4884-92

[62] Chen Y, Vela J, Htoon H, Casson J L, Werder D J, Bussian D A, Klimov V I and Hollingsworth J A 2008 'Giant' multishell CdSe nanocrystal quantum dots with suppressed blinking J. Am. Chem. Soc. 130 5026-7

[63] Bae W K, Padilha L A, Park Y-S, McDaniel H, Robel I, Pietryga J M and Klimov V I 2013 Controlled alloying of the core-shell interface in $\mathrm{CdSe} / \mathrm{CdS}$ quantum dots for suppression of auger recombination ACS Nano 7 3411-9

[64] Park Y-S, Bae W K, Padilha L A, Pietryga J M and Klimov V I 2014 Effect of the core/shell interface on auger recombination evaluated by single-quantum-dot spectroscopy Nano Lett. 14 396-402

[65] Jha P P and Guyot-Sionnest P 2009 Trion decay in colloidal quantum dots ACS Nano 3 1011-5

[66] Ma X, Tan H, Kipp T and Mews A 2010 Fluorescence enhancement, blinking suppression, and gray states of individual semiconductor nanocrystals close to gold nanoparticles Nano Lett. 10 4166-74

[67] Greytak A B, Allen P M, Liu W H, Zhao J, Young E R, Popovic Z, Walker B J, Nocera D G and Bawendi M G 2012 Alternating layer addition approach to $\mathrm{CdSe} / \mathrm{CdS}$ core/shell quantum dots with near-unity quantum yield and high ontime fractions Chem. Sci. 3 2028-34

[68] Chen O et al 2013 Compact high-quality CdSe-CdS coreshell nanocrystals with narrow emission linewidths and suppressed blinking Nat. Mater. 12 445-51

[69] Cui J, Beyler A P, Marshall L F, Chen O, Harris D K, Wanger D D, Brokmann X and Bawendi M G 2013 Direct probe of spectral inhomogeneity reveals synthetic tunability of single-nanocrystal spectral linewidths Nat. Chem. 5 602-6

[70] Tenne R, Teitelboim A, Rukenstein P, Dyshel M, Mokari T and Oron D 2013 Studying quantum dot blinking 
through the addition of an engineered inorganic hole trap ACS Nano 7 5084-90

[71] Hines M A and Guyot-Sionnest P 1996 Synthesis and characterization of strongly luminescing $\mathrm{ZnS}$-Capped CdSe nanocrystals J. Phys. Chem. 100 468-71

[72] Danek M, Jensen K F, Murray C B and Bawendi M G 1996 Synthesis of luminescent thin-film CdSe/ZnSe quantum dot composites using CdSe quantum dots passivated with an overlayer of ZnSe Chem. Mater. 8 173-80

[73] Mekis I, Talapin D V, Kornowski A, Haase M and Weller H 2003 One-pot synthesis of highly luminescent $\mathrm{CdSe} / \mathrm{CdS}$ core/shell nanocrystals via organometallic and 'greener' chemical approaches J. Phys. Chem. B 107 7454-62

[74] Schlamp M C, Peng X G and Alivisatos A P 1997 Improved efficiencies in light emitting diodes made with $\mathrm{CdSe}(\mathrm{CdS})$ core/shell type nanocrystals and a semiconducting polymer J. Appl. Phys. 82 5837-42

[75] Grumbach N, Capek R K, Tilchin E, Rubin-Brusilovski A, Yang J, Ein-Eli Y and Lifshitz E 2015 Comprehensive route to the formation of alloy interface in core/shell colloidal quantum dots J. Phys. Chem. C 119 12749-56

[76] Furdyna J K 1988 Diluted magnetic semiconductors J. Appl. Phys. 64 R29-64

[77] Besombes L, Leger Y, Maingault L, Ferrand D, Mariette $\mathrm{H}$ and Cibert J 2004 Probing the spin state of a single magnetic ion in an individual quantum dot Phys. Rev. Lett. 93207403

[78] Varghese B, Boukari H and Besombes L 2014 Dynamics of a Mn spin coupled to a single hole confined in a quantum dot Phys. Rev. B 9011530775

[79] Lafuente-Sampietro A, Utsumi H, Boukari H, Kuroda S and Besombes L 2016 Spin dynamics of an individual $\mathrm{Cr}$ atom in a semiconductor quantum dot under optical excitation Appl. Phys. Lett. 10953103

[80] Lafuente-Sampietro A, Boukari H and Besombes L 2015 Strain-induced coherent dynamics of coupled carriers and Mn spins in a quantum dot Phys. Rev. B 9281305

[81] Besombes L, Boukari H, Le Gall C, Brunetti A, Cao C L L, Jamet S, Varghese B and Varghese B 2015 Optical control of the spin of a magnetic atom in a semiconductor quantum dot Nanophotonics 4 75-89

[82] Mendes U C, Korkusinski M, Trojnar A H and Hawrylak P 2013 Optical properties of charged quantum dots doped with a single magnetic impurity Phys. Rev. B 88115306

[83] Erwin S C, Zu L, Haftel M I, Efros A L, Kennedy T A and Norris D J 2005 Doping semiconductor nanocrystals Nature 436 91-4

[84] Beaulac R, Schneider L, Archer P I, Bacher G and Gamelin D R 2009 Light-induced spontaneous magnetization in doped colloidal quantum dots Science $\mathbf{3 2 5}$ 973-6

[85] Viswanatha R, Pietryga J M, Klimov V I and Crooker S A 2011 Spin-polarized $\mathrm{Mn}^{\{2+\}}$ emission from Mn-doped colloidal nanocrystals Phys. Rev. Lett. 10767402

[86] Beaulac R, Ochsenbein S T and Gamelin D R 2010 Colloidal transition- metal-doped quantum dots Nanocrystal Quantum Dots ed V I Klimov 2nd edn (Boca Raton, FL: CRC Press) pp 397-453

[87] Govorov A O and Kalameitsev A V 2005 Optical properties of a semiconductor quantum dot with a single magnetic impurity: photoinduced spin orientation Phys. Rev. B 71 35338

[88] Archer P I, Santangelo S A and Gamelin D R 2007 Direct observation of $\mathrm{sp}-\mathrm{d}$ exchange interactions in colloidal $\mathrm{Mn}^{2+}$ - and $\mathrm{Co}^{2+}$-doped CdSe quantum dots Nano Lett. 7 1037-43

[89] Beaulac R, Feng Y, May J W, Badaeva E, Gamelin D R and Li X 2011 Orbital pathways for $\mathrm{Mn}^{+2}$-carrier sp-d exchange in diluted magnetic semiconductor quantum dots Phys. Rev. B 84195324

[90] Barrows C J, Vlaskin V A and Gamelin D R 2015 Absorption and magnetic circular dichroism analyses of giant zeeman splittings in diffusion-doped colloidal $\mathrm{Cd}_{1_{-x}} \mathrm{Mn}_{x} \mathrm{Se}$ quantum dots J. Phys. Chem. Lett. 6 3076-81

[91] Beaulac R, Archer P I, Liu X, Lee S, Salley G M, Dobrowolska M, Furdyna J K and Gamelin D R 2008 Spinpolarizable excitonic luminescence in colloidal $\mathrm{Mn}^{2+}$-doped CdSe quantum dots Nano Lett. 8 1197-201

[92] Brovelli S, Galland C, Viswanatha R and Klimov V I 2012 Tuning radiative recombination in $\mathrm{Cu}$-doped nanocrystals via electrochemical control of surface trapping Nano Lett. 12 4372-9

[93] Beaulac R, Archer P I, Ochsenbein S T and Gamelin D R $2008 \mathrm{Mn}^{2+}$-doped CdSe quantum dots: new inorganic materials for spin-electronics and spin-photonics Adv. Funct. Mater. 18 3873-91

[94] Bussian D A, Crooker S A, Yin M, Brynda M, Efros A L and Klimov V I 2009 Tunable magnetic exchange interactions in manganese-doped inverted core-shell $\mathrm{ZnSe}-\mathrm{CdSe}$ nanocrystals Nat. Mater. $835-40$

[95] Norris D J, Yao N, Charnock F T and Kennedy T A 2001 High-quality manganese-doped ZnSe nanocrystals Nano Lett. 1 3-7

[96] Whitham P J, Knowles K E, Reid P J and Gamelin D R 2015 Photoluminescence blinking and reversible electron trapping in copper-doped CdSe nanocrystals Nano Lett. 15 4045-51

[97] Gerd B, Lars S, Remi B, Paul I A and Daniel R G 2011 Magnetic polaron formation dynamics in $\mathrm{Mn}^{2+}$-doped colloidal nanocrystals up to room temperature $J$. Korean Phys. Soc. 581261

[98] Kłopotowski Ł et al 2011 Magnetic polaron formation and exciton spin relaxation in single $\mathrm{Cd}_{1-X} \mathrm{Mn}_{X} \mathrm{Te}$ quantum dots Phys. Rev. B 8381306

[99] Nelson H D, Bradshaw L R, Barrows C J, Vlaskin V A and Gamelin D R 2015 Picosecond dynamics of excitonic magnetic polarons in colloidal diffusion-doped $\mathrm{Cd}_{1-x} \mathrm{Mn}_{x} \mathrm{Se}$ quantum dots ACS Nano 9 11177-91

[100] Rice W D, Liu W, Baker T A, Sinitsyn N A, Klimov V I and Crooker S A 2015 Revealing giant internal magnetic fields due to spin fluctuations in magnetically doped colloidal nanocrystals Nat. Nanotechnol. 11 137-42

[101] Besombes L, Leger Y, Bernos J, Boukari H, Mariette H, Poizat J P, Clement T, Fernaandez-Rossier J and Aguado R 2008 Optical probing of spin fluctuations of a single paramagnetic $\mathrm{Mn}$ atom in a semiconductor quantum dot Phys. Rev. B 78125324

[102] Bradshaw L R, May J W, Dempsey J L, Li X and Gamelin D R 2014 Ferromagnetic excited-state $\mathrm{Mn}^{2+}$ dimers in $\mathrm{Zn}_{1-x} \mathrm{Mn}_{X}$ Se quantum dots observed by time-resolved magnetophotoluminescence Phys. Rev. B 89115312

[103] Besombes L, Cao C L, Jamet S, Boukari H and Fernandez-Rossier J 2012 Optical control of the spin state of two Mn atoms in a quantum dot Phys. Rev. B 86165306

[104] MØLLER C K 1958 Crystal structure and photoconductivity of cæsium plumbohalides Nature 182 1436-1436

[105] Ishihara T 1994 Optical properties of PbI-based perovskite structures J. Lumin. 60-61 269-74

[106] Hirasawa M, Ishihara T, Goto T, Uchida K and Miura N 1994 Magnetoabsorption of the lowest exciton in perovskite-type compound (CH3NH3)PbI3 Physica B 201 427-30

[107] Zhou H, Chen Q, Li G, Luo S, Song T-B, Duan H-S, Hong Z, You J, Liu Y and Yang Y 2014 Interface engineering of highly efficient perovskite solar cells Science 345 542-6

[108] Kojima A, Teshima K, Shirai Y and Miyasaka T 2009 Organometal halide perovskites as visible-light sensitizers for photovoltaic cells J. Am. Chem. Soc. 131 6050-1 
[109] Lee M M, Teuscher J, Miyasaka T, Murakami T N and Snaith H J 2012 Efficient hybrid solar cells based on mesosuperstructured organometal halide perovskites Science $\mathbf{3 3 8}$ $643-7$

[110] Kim H-S et al 2012 Lead iodide perovskite sensitized allsolid-state submicron thin film mesoscopic solar cell with efficiency exceeding 9\% Sci. Rep. 2591

[111] Jeon N J, Noh J H, Yang W S, Kim Y C, Ryu S, Seo J and Il Seok S 2015 Compositional engineering of perovskite materials for high-performance solar cells Nature $\mathbf{5 1 7}$ 476-80

[112] Mitzi D B, Chondroudis K and Kagan C R 2001 Organicinorganic electronics IBM J. Res. Dev. 45 29-45

[113] Yakunin S, Protesescu L, Krieg F, Bodnarchuk M I, Nedelcu G, Humer M, De Luca G, Fiebig M, Heiss W and Kovalenko M V 2015 Low-threshold amplified spontaneous emission and lasing from colloidal nanocrystals of caesium lead halide perovskites Nat. Commun. 68056

[114] Zhu H, Fu Y, Meng F, Wu X, Gong Z, Ding Q, Gustafsson M V, Trinh M T, Jin S and Zhu X-Y 2015 Lead halide perovskite nanowire lasers with low lasing thresholds and high quality factors Nat. Mater. 14 636-42

[115] Fang Y, Dong Q, Shao Y, Yuan Y and Huang J 2015 Highly narrowband perovskite single-crystal photodetectors enabled by surface-charge recombination Nat. Photon. 9 679-86

[116] Sutherland B R, Johnston A K, Ip A H, Xu J, Adinolfi V, Kanjanaboos P and Sargent E H 2015 Sensitive, fast, and stable perovskite photodetectors exploiting interface engineering ACS Photonics 2 1117-23

[117] Park Y-S, Guo S, Makarov N S and Klimov V I 2015 Room temperature single-photon emission from individual perovskite quantum dots ACS Nano 9 10386-93

[118] Kepenekian M, Robles R, Katan C, Sapori D, Pedesseau L and Even J 2015 Rashba and dresselhaus effects in hybrid organic-inorganic perovskites: from basics to devices ACS Nano 9 11557-67

[119] Zheng F, Tan L Z, Liu S and Rappe A M 2015 Rashba spinorbit coupling enhanced carrier lifetime in $\mathrm{CH}_{3} \mathrm{NH}_{3} \mathrm{PbI}_{3}$ Nano Lett. 15 7794-800

[120] Dirin D N, Dreyfuss S, Bodnarchuk M I, Nedelcu G, Papagiorgis P, Itskos G and Kovalenko M V 2014 Lead halide perovskites and other metal halide complexes as inorganic capping ligands for colloidal nanocrystals $J$. Am. Chem. Soc. 136 6550-3

[121] Nedelcu G, Protesescu L, Yakunin S, Bodnarchuk M I, Grotevent M J and Kovalenko M V 2015 Fast anionexchange in highly luminescent nanocrystals of cesium lead halide perovskites $\left(\mathrm{CsPbX}_{3}, \mathrm{X}=\mathrm{Cl}, \mathrm{Br}, \mathrm{I}\right)$ Nano Lett. 15 5635-40

[122] Umebayashi T, Asai K, Kondo T and Nakao A 2003 Electronic structures of lead iodide based low-dimensional crystals Phys. Rev. B 67155405

[123] Brandt R E, Stevanović V, Ginley D S and Buonassisi T 2015 Identifying defect-tolerant semiconductors with high minority-carrier lifetimes: beyond hybrid lead halide perovskites MRS Commun. 5 265-75

[124] Even J, Pedesseau L and Katan C 2014 Analysis of multivalley and multibandgap absorption and enhancement of free carriers related to exciton screening in hybrid perovskites J. Phys. Chem. C 118 11566-72

[125] Tanaka K, Takahashi T, Ban T, Kondo T, Uchida K and Miura N 2003 Comparative study on the excitons in leadhalide-based perovskite-type crystals $\mathrm{CH}_{3} \mathrm{NH}_{3} \mathrm{PbBr}_{3}$ CH3NH3PbI3 Solid State Commun. 127 619-23

[126] Pedesseau L, Kepenekian M, Robles R, Sapori D, Katan C and Even J 2016 Theoretical studies of Rashba and Dresselhaus effects in hybrid organiciInorganic perovskites for optoelectronic applications Proc. SPIE 9742 97421B
[127] Vaxenburg R, Lifshitz E and Efros A L 2013 Suppression of auger-stimulated efficiency droop in nitride-based light emitting diodes Appl. Phys. Lett. 102031120

[128] Vaxenburg R, Rodina A, Lifshitz E and Efros A L 2013 The role of polarization fields in auger-induced efficiency droop in nitride-based light-emitting diodes Appl. Phys. Lett. 103221111

[129] Kloper V, Osovsky R, Kolny-Olesiak J, Sashchiuk A and Lifshitz E 2007 The growth of colloidal cadmium telluride nanocrystal quantum dots in the presence of $\mathrm{Cd} 0$ nanoparticles J. Phys. Chem. C 111 10336-41

[130] Osovsky R, Cheskis D, Kloper V, Sashchiuk A, Kroner M and Lifshitz E 2009 Continuous-wave pumping of multiexciton bands in the photoluminescence spectrum of a single CdTe-CdSe core-shell colloidal quantum dot Phys. Rev. Lett. 102197401

[131] Bacher G 2003 Optical spectroscopy on epitaxially grown IIVI single quantum dots Single Quantum Dots (topics in Applied Physics) vol 90 (Berlin/Heidelberg: Springer) $147-84$

[132] Nozik A J 2002 Quantum dot solar cells Physica E 14 115-20

[133] Tilchin J, Rabouw F T, Isarov M, Vaxenburg R, Van Dijk-Moes R J A, Lifshitz E and Vanmaekelbergh D 2015 Quantum confinement regimes in CdTe nanocrystals probed by single dot spectroscopy: from strong confinement to the bulk limit ACS Nano $97840-5$

[134] Grumbach N, Rubin-Brusilovski A, Maikov G I, Tilchin E and Lifshitz E 2013 Manipulation of carrier-Mn ${ }^{2+}$ exchange interaction in $\mathrm{CdTe} / \mathrm{CdSe}$ colloidal quantum dots by controlled positioning of $\mathrm{Mn}^{2+}$ impurities J. Phys. Chem. C 117 21021-7

[135] Grumbach N, Rubin-Brusilovski A, Maikov G I, Tilchin E and Lifshitz E 2013 Manipulation of carrier-Mn ${ }^{2+}$ exchange interaction in $\mathrm{CdTe} / \mathrm{CdSe}$ colloidal quantum dots by controlled positioning of $\mathrm{Mn}^{2+}$ impurities J. Phys. Chem. C 117 21021-7

[136] Furdyna J K 1988 Diluted magnetic semiconductors J. Appl. Phys. 64 R29-64

[137] Isarov M, Tan L Z, Bodnarchuk M I, Kovalenko M V, Rappe A M and Lifshitz E 2017 Rashba effect in a single colloidal $\mathrm{CsPbBr} 3$ perovskite nanocrystal detected by magneto-optical measurements Nano Lett. 17 5020-6

[138] Protesescu L, Yakunin S, Bodnarchuk M I, Krieg F, Caputo R, Hendon C H, Yang R X, Walsh A and Kovalenko M V 2015 Nanocrystals of cesium lead halide perovskites $(\mathrm{CsPbX} 3, \mathrm{X}=\mathrm{Cl}, \mathrm{Br}$, and $\mathrm{I})$ : novel optoelectronic materials showing bright emission with wide color gamut Nano Lett. 15 3692-6

[139] Tilchin J, Dirin D N, Maikov G I, Sashchiuk A, Kovalenko M V and Lifshitz E 2016 Hydrogen-like wannier-mott excitons in single crystal of methylammonium lead bromide perovskite ACS Nano 10 6363-71

[140] Buin A, Comin R, Xu J, Ip A H and Sargent E H 2015 Halide-dependent electronic structure of organolead perovskite materials Chem. Mater. 27 4405-12

[141] Stranks S D, Burlakov V M, Leijtens T, Ball J M, Goriely A and Snaith H J 2014 Recombination kinetics in organic-inorganic perovskites: excitons, free charge, and subgap states Phys. Rev. Appl. 234007

[142] Wu X, Trinh M T, Niesner D, Zhu H, Norman Z, Owen J S, Yaffe O, Kudisch B J and Zhu X-Y 2015 Trap states in lead iodide perovskites J. Am. Chem. Soc. 137 2089-96

[143] van Bree J, Silov A Y, Koenraad P M, Flatté M E and Pryor C E $2012 \mathrm{~g}$ factors and diamagnetic coefficients of electrons, holes, and excitons in InAs/InP quantum dots Phys. Rev. B 85165323

[144] Jovanov V et al 2012 Highly nonlinear excitonic zeeman spin splitting in composition-engineered artificial atoms Phys. Rev. B 85165433 
[145] Manser J S, Christians J A and Kamat P V 2016 Intriguing optoelectronic properties of metal halide perovskites Chem. Rev. 116 12956-3008

[146] Yang J and Kelly T L 2017 Decomposition and cell failure mechanisms in lead halide perovskite solar cells Inorg. Chem. 56 92-101

[147] Filip M R, Eperon G E, Snaith H J and Giustino F 2014 Steric engineering of metal-halide perovskites with tunable optical band gaps Nat. Commun. 55757

[148] Tan L Z, Zheng F and Rappe A M 2017 Intermolecular interactions in hybrid perovskites understood from a combined density functional theory and effective hamiltonian approach ACS Energy Lett. 2 937-42

[149] Yaffe O et al 2017 Local polar fluctuations in lead halide perovskite crystals Phys. Rev. Lett. 118136001

[150] Brivio F, Butler K T, Walsh A and van Schilfgaarde M 2014 Relativistic quasiparticle self-consistent electronic structure of hybrid halide perovskite photovoltaic absorbers Phys. Rev. B 89155204

[151] Leguy A M A et al 2015 The dynamics of methylammonium ions in hybrid organic-inorganic perovskite solar cells Nat. Commun. 67124

[152] Stoumpos C C, Malliakas C D and Kanatzidis M G 2013 Semiconducting tin and lead iodide perovskites with organic cations: phase transitions, high mobilities, and near-infrared photoluminescent properties Inorg. Chem. 52 9019-38

[153] Weller M T, Weber O J, Henry P F, Di Pumpo A M and Hansen T C 2015 Complete structure and cation orientation in the perovskite photovoltaic methylammonium lead iodide between 100 and 352 K Chem. Commun. 51 4180-3

[154] Poglitsch A and Weber D 1987 Dynamic disorder in methylammoniumtrihalogenoplumbates (II) observed by millimeter-wave spectroscopy J. Chem. Phys. 87 6373-8

[155] Bakulin A A et al 2015 Real-time observation of organic cation reorientation in methylammonium lead iodide perovskites J. Phys. Chem. Lett. 6 3663-9
[156] Frost J M, Butler K T, Brivio F, Hendon C H, van Schilfgaarde M and Walsh A 2014 Atomistic origins of high-performance in hybrid halide perovskite solar cells Nano Lett. 14 2584-90

[157] Bertolotti F et al 2016 Crystal symmetry breaking and vacancies in colloidal lead chalcogenide quantum dots $\mathrm{Nat}$. Mater. 15 987-94

[158] Bychkov Y A and Rashba E I 1984 Properties of a 2D electron gas with lifted spectral degeneracy JETP Lett. 3978

[159] Etienne T, Mosconi E and De Angelis F 2016 Dynamical origin of the rashba effect in organohalide lead perovskites: a key to suppressed carrier recombination in perovskite solar cells? J. Phys. Chem. Lett. 7 1638-45

[160] Manchon A, Koo H C, Nitta J, Frolov S M and Duine R A 2015 New perspectives for rashba spin-orbit coupling Nat. Mater. 14 871-82

[161] Ganichev S D and Golub L E 2014 Interplay of rashba/ dresselhaus spin splittings probed by photogalvanic spectroscopy -a review Phys. Status Solidi 251 1801-23

[162] Rashba E I and Sherman E Y 1988 Spin-orbital band splitting in symmetric quantum wells Phys. Lett. A 129 175-9

[163] Dresselhaus G 1955 Spin-orbit coupling effects in zinc blende structures Phys. Rev. 100580

[164] Stroppa A, D Sante D, Barone P, Bokdam M, Kresse G, Franchini C, Whangbo M-H and Picozzi S 2014 Tunable ferroelectric polarization and Its interplay with spin-orbit coupling in tin iodide perovskites Nat. Commun. 55900

[165] Yarita N, Tahara H, Ihara T, Kawawaki T, Sato R, Saruyama M, Teranishi T and Kanemitsu Y 2017 Dynamics of charged excitons and biexcitons in $\mathrm{CsPBr}_{3}$ perovskite nanocrystals revealed by femtosecond transient-absorption and single-dot luminescence spectroscopy J. Phys. Chem. Lett. 8 1413-8

[166] Fu M, Tamarat P, Huang H, Even J, Rogach A L and Lounis B 2017 Neutral and charged exciton fine structure in single lead halide perovskite nanocrystals revealed by magneto-optical spectroscopy Nano Lett. 17 2895-901 